\title{
ArcheoSciences
}

Revue d'archéométrie

$36 \mid 2012$

Varia

\section{Étude archéométallurgique du dépôt de grands bronzes du sanctuaire gallo-romain du Vieil- Evreux (Eure)}

Archaeometallurgical Study of the Large Bronze Hoard Discovered on the GalloRoman Sanctuary of Le Vieil-Evreux (Eure)

Aurélia Azéma, Benoît Mille, Fabien Pilon, Jean-Claude Birolleau et Laurent Guyard

\section{OpenEdition}

Journals

Édition électronique

URL : https://journals.openedition.org/archeosciences/3670

DOI : 10.4000/archeosciences.3670

ISBN : 978-2-7535-2243-5

ISSN : 2104-3728

Éditeur

Presses universitaires de Rennes

Édition imprimée

Date de publication : 31 décembre 2012

Pagination : 153-173

ISBN : 978-2-7535-2241-1

ISSN : 1960-1360

Référence électronique

Aurélia Azéma, Benoît Mille, Fabien Pilon, Jean-Claude Birolleau et Laurent Guyard, «Étude archéométallurgique du dépôt de grands bronzes du sanctuaire gallo-romain du Vieil-Evreux (Eure) », ArcheoSciences [En ligne], 36 | 2012, mis en ligne le 30 décembre 2014, consulté le 31 janvier 2022. URL : http://journals.openedition.org/archeosciences/3670; DOI : https://doi.org/10.4000/ archeosciences.3670 


\title{
Étude archéométallurgique du dépôt de grands bronzes du sanctuaire gallo-romain du Vieil-Évreux (Eure)
}

\author{
Archaeometallurgical Study of the Large Bronze Hoard Discovered \\ on the Gallo-Roman Sanctuary of Le Vieil-Evreux (Eure)
}

\author{
Aurélia AzÉma*, Benoît Mille*,**, Fabien Pilon***, \\ Jean-Claude Birolleau*** et Laurent GuYARD****
}

\begin{abstract}
Résumé : Cet article présente les résultats d’une étude archéométallurgique réalisée sur un important dépôt de bronze découvert en 1840 sur le site du sanctuaire gallo-romain du Vieil-Evreux. De récentes découvertes de fragments au sein du sanctuaire, ainsi qu’une relecture des données d'archives, ont amené à redéfinir la nature et le contexte de ce dépôt.

Les travaux ont été exécutés au C2RMF et au CEA Le Ripault, en collaboration avec la MADE. L'étude a impliqué l'utilisation de techniques multiples, telles que la radiographie, la tomographie, l'endoscopie, ou encore la détermination de la composition élémentaire par ICP-AES des différentes pièces de bronze.

Deux principaux résultats ont été obtenus à l'issue de cette étude archéométrique. Le premier est directement lié à la compréhension du site du Vieil-Évreux par la détermination du nombre minimal de statues constituant le dépôt, et par une identification plus précise des statues. Le second, plus général, consiste à intégrer et à comparer les données acquises au Vieil-Evreux au sein du vaste programme de recherche consacré à l'étude des techniques de fabrication de la grande statuaire antique en bronze (notamment l'étude du procédé indirect de fonte en creux à la cire perdue et de l'assemblage par soudage).
\end{abstract}

\begin{abstract}
This article reports the results of an archeometallurgical study carried out on an important bronze hoard discovered in 1840 on the gallo-roman sanctuary of Le Vieil-Evreux. Recent excavations provided other fragments, and a study of the archive data, led to redefine the nature and context of this hoard. The work was conducted by the C2RMF, in collaboration with the CEA Le Ripault and the MADE, and the analytical procedure involved the use of multiple techniques such as radiography, tomography, endoscopy, or the determination of elemental composition by ICP-AES of the various pieces of bronze. Two main results were obtained so far. The first is directly related to the understanding of the Vieil-Evreux site by determining the minimal number of statues in the bronze hoard and more accurate the identification of their iconography. The second, more general, is to integrate and compare the data in the research program concerning the study of the manufacturing techniques of the Greek and Roman large bronze statues (including the study of the lost wax process and the welding process).
\end{abstract}

Mots clé : grande statuaire, bronze, gallo-romain, cire perdue, soudage par fusion au bronze liquide, composition élémentaire.

Keywords: large bronze statuary, gallo-roman, lost wax casting, flow fusion welding, bulk metal analysis.

\footnotetext{
* Centre de Recherche et de Restauration des Musées de France (C2RMF), UMR171 CNRS - ministère de la Culture, Palais du Louvre - porte des Lions, 14, quai François-Mitterrand, 75001 Paris.

** Préhistoire et Technologie, UMR7055 CNRS - Université de Paris X Nanterre, Maison René-Ginouvès, Archéologie et Ethnologie - 21, allée de l'Université, 92023 Nanterre Cedex.

*** CEA, DAM, Le Ripault, 37260, Monts, France.

**** Mission Archéologique Départementale de l'Eure (MADE), Site archéologique de Gisacum, 8, rue des thermes 27930 Le Vieil-Evreux.
} 


\section{INTRODUCTION}

Un important dépôt de bronze a été découvert en 1840 lors des premières fouilles réalisées sur le site de la ville sanctuaire gallo-romaine du Vieil-Evreux. Ce mobilier, aujourd'hui conservé au Musée municipal d'Evreux, comprend plus d'une centaine de fragments issus d'une ou plusieurs statues monumentales, une remarquable statue de Jupiter $(92 \mathrm{~cm})$, une statue d'Apollon $(69 \mathrm{~cm})$, un fragment de plaque gravée d'une inscription $\left(41 \times 60 \mathrm{~cm}^{2}\right)$, un fragment de corniche $\left(14,5 \times 74,5 \mathrm{~cm}^{2}\right.$ ), et quatre bras (échelle $1 / 4$ et $1 / 2$ ) (figure 1 ). Les fouilles du site gallo-romain ont aujourd'hui repris sous la direction de la Mission Archéologique Départementale de l'Eure (MADE). La découverte de nouveaux fragments au sein du sanctuaire, ainsi qu'une relecture des données d'archives, invitent à réactualiser l'interprétation de la nature et du contexte de ce dépôt. L'abandon des statues semble en effet correspondre à un acte spécifique qui pourrait avoir marqué la fermeture du sanctuaire lors de sa transformation en site fortifié (castellum) vers 275 de notre ère (Guyard et Bertaudière, 2007).
Une étude archéométallurgique a été réalisée dans le cadre d'un projet de recherche conduit par le C2RMF, consacré à l'étude des techniques de fabrication de la grande statuaire antique en bronze. Cette étude poursuit plusieurs objectifs :

- légitimer l'existence d'un lien entre les découvertes du $\mathrm{XIX}^{\mathrm{e}}$ et celles du XXI ${ }^{\mathrm{e}}$ siècle. Les récentes découvertes étant très bien contextualisées, cela permettrait de reconstituer une bonne partie de l'histoire de ces découvertes anciennes (par exemple la place originelle des statues dans le sanctuaire, la date de leur mise en place, etc.);

- rechercher les associations entre fragments afin de déterminer le nombre minimal de statues sur la base de considérations technologiques et de la composition élémentaire du métal (en particulier pour ce qui concerne le nombre de statues monumentales);

- aider à la reconstitution iconographique des statues représentées sous la forme de fragments.

- confronter la technique de fabrication des grands bronzes du Vieil-Evreux avec celle de statues comparables découvertes dans le monde romain, notamment pour la question des statues monumentales.
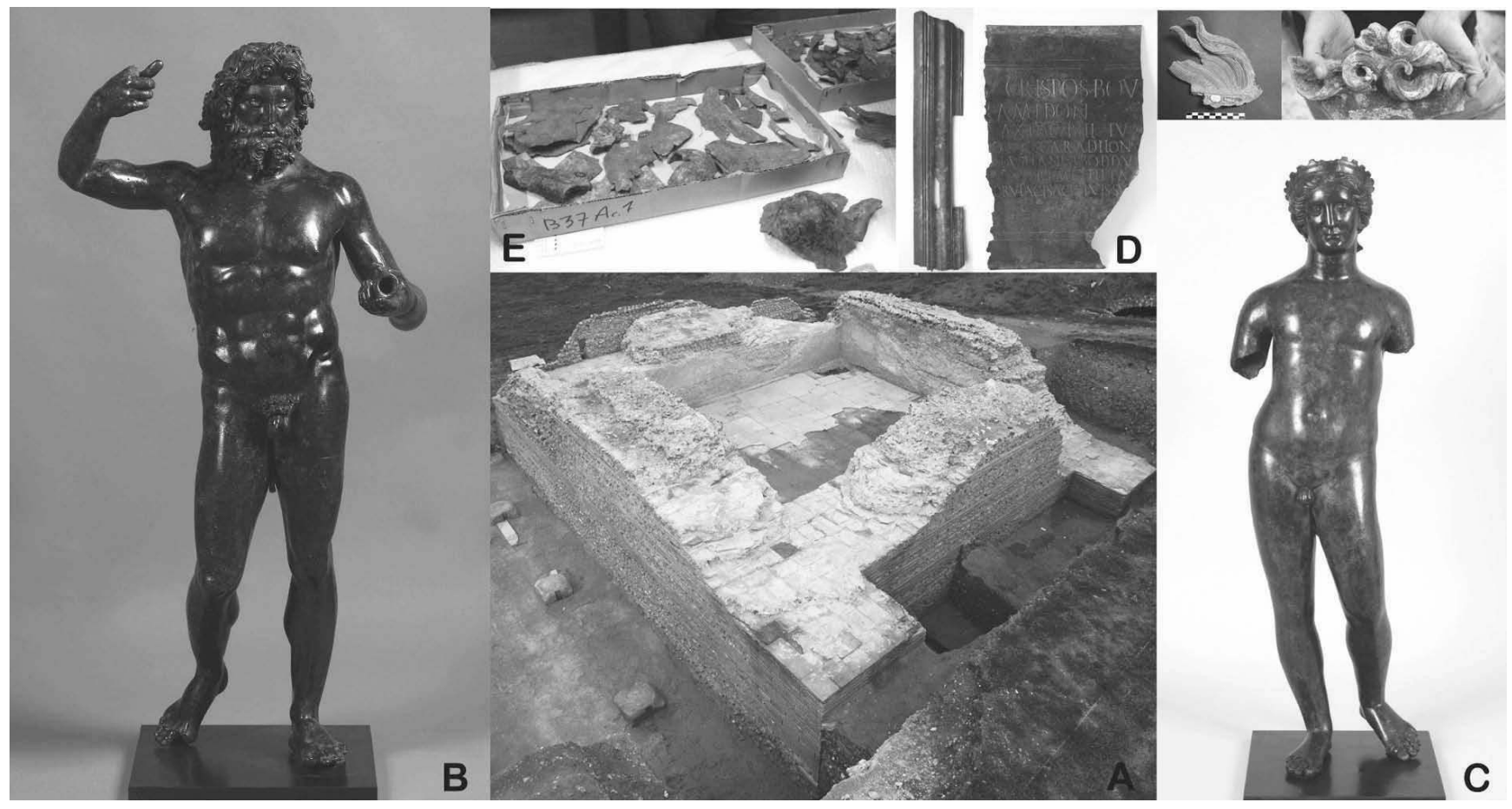

Figure 1 : Le dépôt de bronze du site gallo-romain du Vieil-Evreux : cella centrale du grand sanctuaire (A) CMADE, statue de Jupiter (B) (C) D. Bagault C2RMF, statue d'Apollon (C), le fragment de plaque et sa corniche (D) (C) A. Chauvet C2RMF, l'ensemble des fragments monumentaux (E) (C) B. Mille C2RMF.

Figure 1: The bronze hoard of the gallo-roman sanctuary of le Vieil-Evreux : cella of the great sanctuary (A) (CMADE, Jupiter statue, (C) D. Bagault C2RMF, Apollo statue (C), a slab fragment and its cornice (D) (C) A. Chauvet C2RMF, fragments of a monumental bronze statue (E) (C) B. Mille C2RMF. 


\section{Mise aU Point d'Une STRATÉGie ANALYTIQUe}

\section{La technique de fabrication des grands bronzes}

L'un des principaux acquis de notre recherche a été de démontrer que la fabrication des statues antiques en bronze (du vi siècle avant J.-C. au ve siècle après J.-C.) reposait sur la maîtrise de deux techniques métallurgiques complexes : le procédé indirect de fonte en creux à la cire perdue qui permettait d'obtenir les différentes parties dissociées de la statue (tête, jambe, bras, corps...) et le soudage par fusion au bronze liquide qui les assemblait (Bouquillon et al., 2006; Mille, 2007; Darblade-Audoin et Mille, 2008). La technique d'assemblage consistait à verser du bronze liquide entre les deux pièces à assembler afin de provoquer le soudage par fusion partielle de leur bord et ainsi à former un joint par continuité de la matière (Azéma et Mille, 2010; Azéma et Mille, 2012). Les soudures se présentent sous forme de cordons linéaires, souvent ponctués d'élargissements en forme de cuvettes. Nous avons de cette manière identifié des marqueurs technologiques observables sur les statues, permettant de donner une description détaillée des étapes et des méthodes de sa fabrication. Ainsi, la façon de travailler la cire (en particulier les épaisseurs des parois), le plan de coulée (emplacement des coupes et nombre de pièces coulées), la nature de l'alliage utilisé pour effectuer ces première coulées (qualifiées de coulées primaires), les emplacements et les dimensions des armatures et des clous distanciateurs (ils servaient à renforcer et à empêcher le noyau de se déplacer dans le moule lors de la coulée), le mode de préparation des pièces avant soudage, le détail de la technique de soudage et la composition du métal déposé par le biais de coulées secondaires d'assemblage, et même la manière de corriger ou dissimuler les défauts de coulée (réparure), sont autant de critères qui permettent de définir la technique de fabrication d'une statue. Ces marqueurs technologiques peuvent ainsi mettre en évidence certaines évolutions des techniques de fabrication des grands bronzes, révéler l'existence de différentes écoles de fondeurs, voire même aider à identifier la main spécifique d'un artisan. Ces marqueurs se révèlent également utiles pour tester l'existence de liens entre différentes statues d'un même ensemble, comme dans le cas précis des objets et fragments constituant le dépôt de bronze du Vieil-Evreux.

\section{Étude technologique : radiographies $\mathrm{X}$, endoscopie, relevés}

Ce protocole d'étude permet, dans un premier temps, d'établir une cartographie plus précise de l'objet (nombre de coulées primaires, emplacements de clous distanciateurs, localisation des réparations et des zones d'assemblage, mesure des épaisseurs de parois, etc.) afin de distinguer les étapes de sa fabrication. Il se base sur la réalisation d'une couverture radiographique complète (radiographies X effectuées suivant plusieurs angles et des conditions d'exposition variées), éventuellement complétée par des examens endoscopiques. La figure 5 rassemble les observations et relevés effectués sur les statues d'Apollon et de Jupiter, ainsi que sur la plaque gravée et deux des fragments de bronze monumental.

\section{Analyse de la composition élémentaire du métal par ICP-AES sur micro-prélèvements}

La composition élémentaire du dépôt de bronze a été déterminée sur prélèvements par échantillonnage des différentes pièces qui le constituent. Ils sont localisés de façon à obtenir systématiquement une information sur les alliages employés pour chaque coulée primaire, coulée secondaire d'assemblage et coulée secondaire de réparation. Au total, une centaine de prélèvements ont été effectués par microforages de $1 \mathrm{~mm}$ de diamètre environ afin de récolter des copeaux de métal sain. Un nettoyage sous loupe binoculaire a permis d'éliminer les éventuelles traces restantes d'oxydes et autres pollutions qui pourraient avoir une influence sur les analyses qui ont suivi. Les prélèvements ont été mis en solution dans l'eau régale et analysés par la technique ICP-AES (Inductively Coupled Plasma - Atomic Emission Spectrometry), en nébulisant les solutions obtenues au sein d'un plasma d'argon produit par couplage inductif, et en mesurant le spectre d'émission atomique qui en résulte (le protocole d'analyse est détaillé dans Bourgarit et Mille, 2003). Les analyses élémentaires ont été effectuées par le CEA Le Ripault sur un spectromètre à plasma à couplage inductif Activa de Horiba Jobin Yvon doté d'une focale de $640 \mathrm{~mm}$, de deux réseaux pour la dispersion en longueur d'onde (4343 traies/ $\mathrm{mm}$ et 2400 traies $/ \mathrm{mm}$ ) et d'un détecteur CCD de $1 \mathrm{mega}-$ pixel. Le tableau de résultats est présenté en annexe; les incertitudes sont de $4 \%$ relatifs pour les éléments majeurs et de $10 \%$ relatifs pour les impuretés.

\section{RÉSUlTATS-DISCUSSION}

\section{Un dépôt hétérogène}

En première approche, les résultats révèlent une importante diversité aussi bien en ce qui concerne la composition élémentaire des alliages employés que la technique de 
fabrication. Quelques considérations très simples permettent de distinguer quatre ensembles nettement différents que nous décrirons tout d'abord séparément. Nous procèderons ensuite à une analyse globale de l'ensemble du dépôt, en particulier dans le but de déterminer le nombre minimal de statues qui le constituent.

\section{Les fragments de plaque et de corniche}

Les analyses élémentaires confirment le lien entre le fragment de plaque et le fragment de corniche : la corniche a été coulée avec le même métal que celui qui a permis d'assembler par soudage les différentes parties de la plaque gravée : un bronze au plomb (8 à $10 \%$ d'étain, $10 \%$ de plomb) dont le cortège d'impuretés est identique (figure 5 , Annexe). Compte tenu de la présence d'une coulée secondaire d'assemblage sur toute la longueur d'un bord vertical (observable en radiographie) et d'un texte gravé incomplet, la plaque entière devait être constituée d'au moins trois pièces. Nous ajouterons que des analyses sur l'accélérateur
AGLAE (Accélérateur Grand Louvre d'Analyse Elémentaire) ont indiqué la présence de cinabre $(\mathrm{HgS})$ remplissant le creux des lettres du texte gravé. Lors de la restauration, il a cependant été montré que ce remplissage est probablement moderne (Pariselle, 2011).

\section{La statue d'Apollon}

La statue d'Apollon $(0,69 \mathrm{~m})$, datée du $\mathrm{II}^{\mathrm{e}}$ siècle après J.-C., résulte, de l'assemblage par soudage de cinq pièces principales (tête, corps + jambe droite, deux bras aujourd'hui disparus, jambe gauche) obtenues par le procédé indirect de fonte en creux à la cire perdue (coulées primaires). Le sexe constitue un cas particulier : il n'est porté que par la jambe droite. Cette anomalie anatomique ne s'explique que si le sexe a été coulé en même temps que l'ensemble corps + jambe droite. Il aurait alors été directement sculpté en cire, puis rapporté sur la jambe. Par ailleurs, au moins deux pièces ont été élaborées par coulée secondaire : la couronne et les mèches de cheveux retombant du chignon ont été directe-
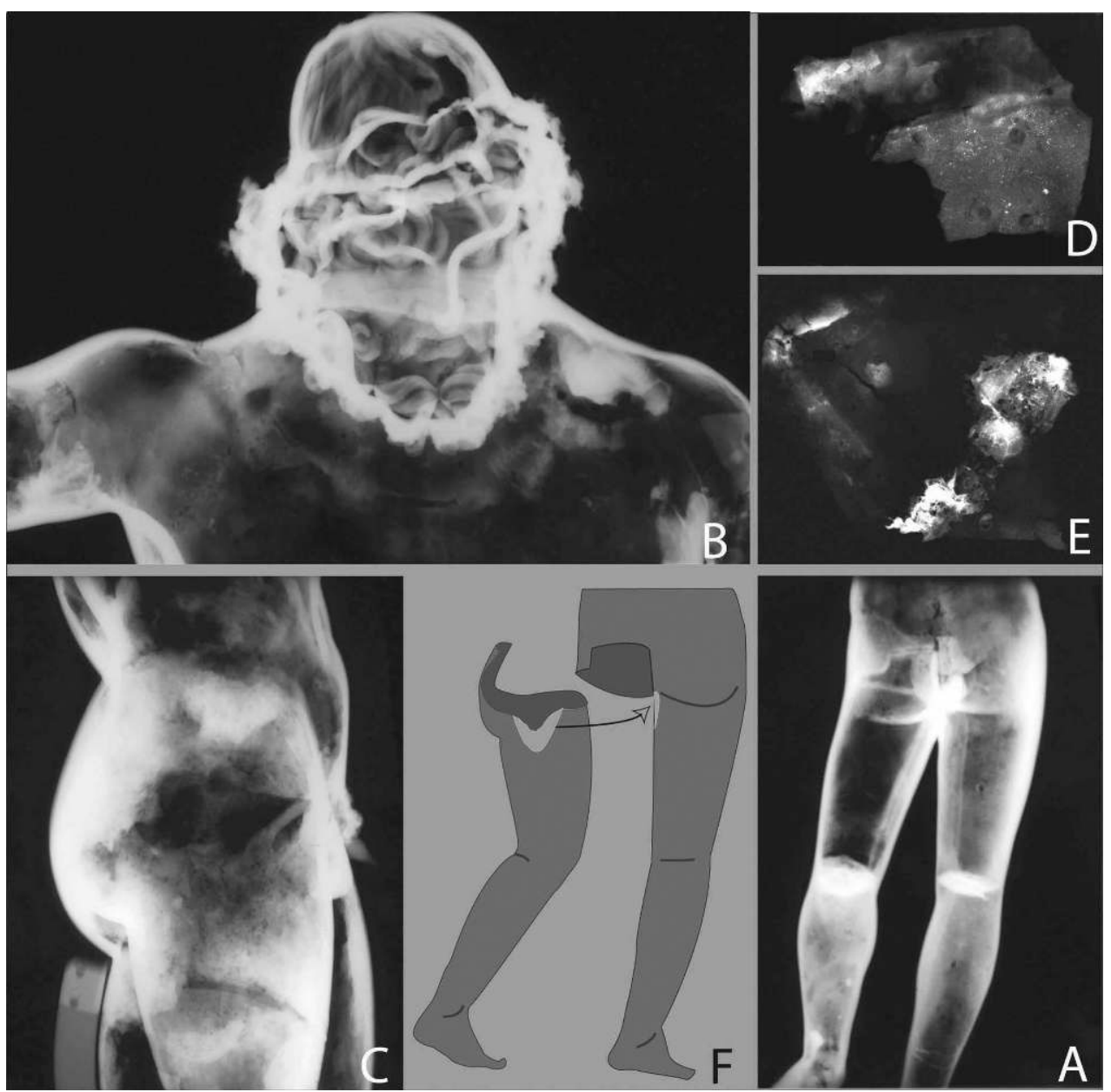

Figure 2 : Radiographies X des jambes d'Apollon (A), du buste (B) et des jambes (C) de Jupiter, et des fragments C2RMF69362 (D) et C2RMF69516 (E) CT. Borel C2RMF, schéma de la jonction entre la jambe gauche et le corps d'Apollon (F) (C) A. Azéma C2RMF.

Figure 2: $A$ to E: $X$-ray radiographs. Apollo statue (A), chest (B) and legs $(C)$ of the Jupiter statue, fragments of C2RMF69362 (D) and C2RMF69516 (E) (C) T. Borel C2RMF. F: drawing of the join between the left leg and the body of Apollo (C) A. Azéma C2RMF. 
ment sculptées en cire et ajoutées sur la statue en bronze. Ce procédé permet de former les pièces et de les assembler sur le corps en un seul jet de métal; il est également attesté sur le cheval de Neuvy-en-Sullias, au moins pour la réalisation du toupet au sommet de la tête (Mille, 2007). Le découpage décrit ci-dessus est reporté sur la figure 5 . Les coulées primaires sont faites d'un bronze au plomb (teneur moyenne Sn : 7,8 $\sigma 0,9 \% \mathrm{~m}, \mathrm{~Pb}: 15,2 \sigma 2 \% \mathrm{~m})$ dont le cortège d'impuretés est relativement homogène. La composition du sexe diffère quelque peu des autres coulées primaires, en particulier par sa composition plus élevée en plomb ( $\mathrm{Sn}$ : $7,9 \% \mathrm{~m}, \mathrm{~Pb}: 22,4 \% \mathrm{~m})$, qu'il faut sans doute imputer à un phénomène de ségrégation du plomb au moment de la solidification de cette zone, plus épaisse qu'ailleurs. La composition du bronze employé pour les éléments rapportés par coulée secondaire s'apparente à celle du métal utilisé pour le soudage. Ainsi, la mèche du chignon, également riche en plomb ( $\mathrm{Sn}: 7,0 \% \mathrm{~m}, \mathrm{~Pb}: 20,1 \% \mathrm{~m})$, révèle un cortège d'impuretés dont les teneurs sont différentes de celles de la coulée primaire mais identiques à celles des coulées secondaires d'assemblage ( $\mathrm{Zn}, \mathrm{Fe}, \mathrm{S})$. Nous soulignerons enfin la similitude des proportions en éléments majeurs du métal de soudure (teneur moyenne Sn : 7,1 $\sigma$ 0,5\%m, $\mathrm{Pb}: 14,9 \sigma 0,6 \% \mathrm{~m})$ avec celles des coulées primaires. Les clous distanciateurs (1,8 $\mathrm{mm}$ de côté) ont été régulièrement répartis sur l'ensemble de la statue. En plus des clous, il existait également des armatures de section carrée $(4,5 \mathrm{~mm}$ de côté), qui ont été retirées après la coulée. Compte tenu de leur emplacement, nous pouvons émettre l'hypothèse de la présence de six armatures traversant horizontalement la statue (mollet-tibia de droite et de gauche, cuisse avant-arrière de droite et de gauche, ventre-bas du dos et cou-nuque) et d'une verticale allant de la tête au pied soclé. Les clous distanciateurs sont le dispositif habituel pour maintenir en place le noyau dans le moule au moment de la coulée des grands bronzes antiques. Une armature verticale est quant à elle souvent utilisée pour la fabrication de grandes statuettes en creux par le procédé direct de fonte à la cire perdue, servant alors de squelette pour l'élaboration du noyau et remplaçant les clous distanciateurs (voir par exemple la statuette d'homme au sagum du dépôt de Neuvy-en-Sullias, Mille et Borel, 2007). Quant à la présence conjointe d'armatures et de clous distanciateurs, elle n'est documentée que pour les statues de très grande dimension, les armatures ayant alors pour fonction de renforcer la structure du noyau (Janietz Schwarz et Rouiller, 1996). Le cas du moyen bronze du Vieil-Evreux est donc atypique, et la fonction des armatures - en particulier horizontales - reste inexpliquée. La présence de gerces (infiltrations de métal dans le noyau interne) au niveau du cou, des genoux et des chevilles est l'indication
Figure 3 : Statue d'Apollon; observation d'une cuvette d'assemblage par soudure du bras gauche sur le corps. (C) A. Chauvet C2RMF.

Figure 3: Apollo statue; a fusion welding basin between the left arm and the body (C) A. Chauvet C2RMF.

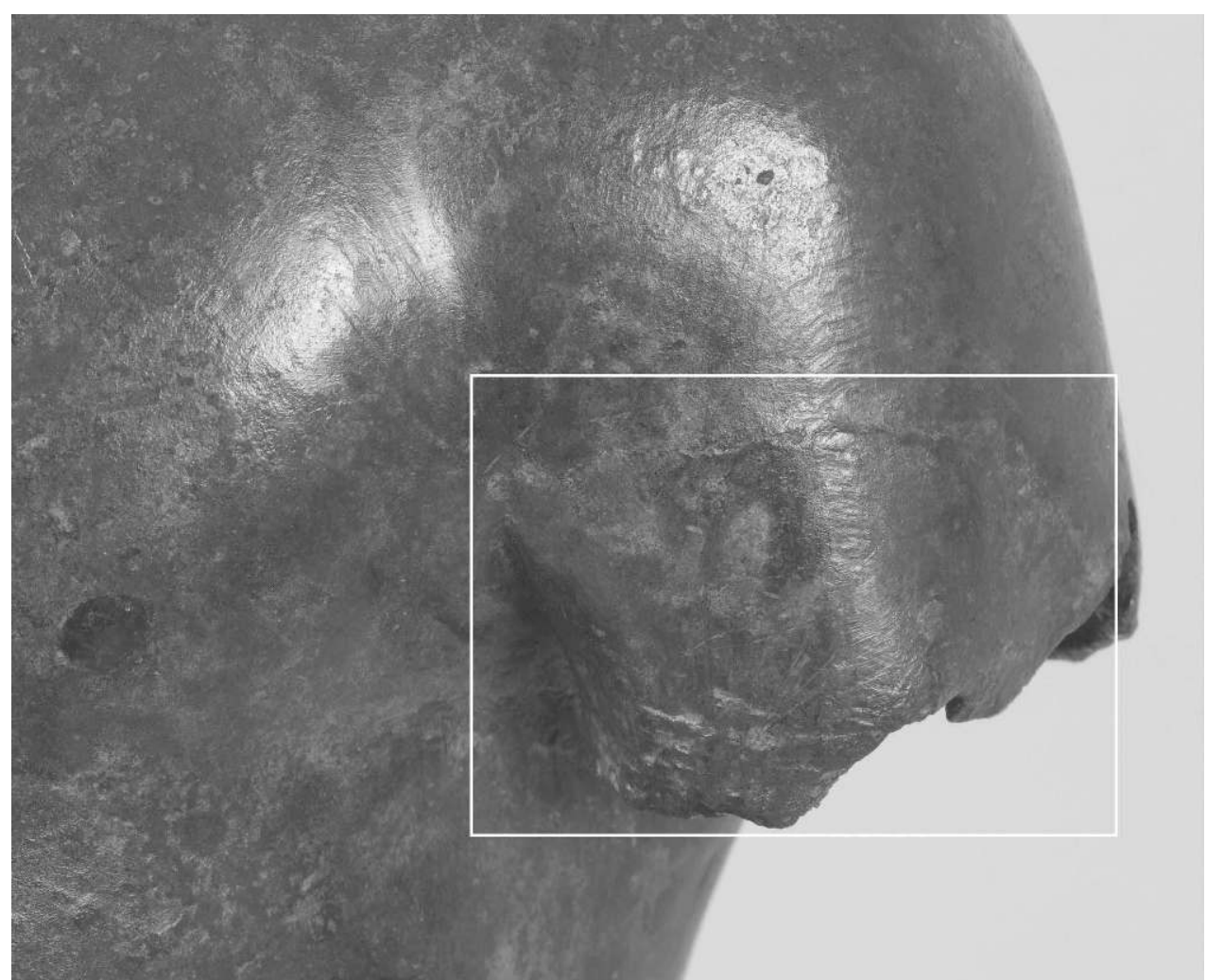




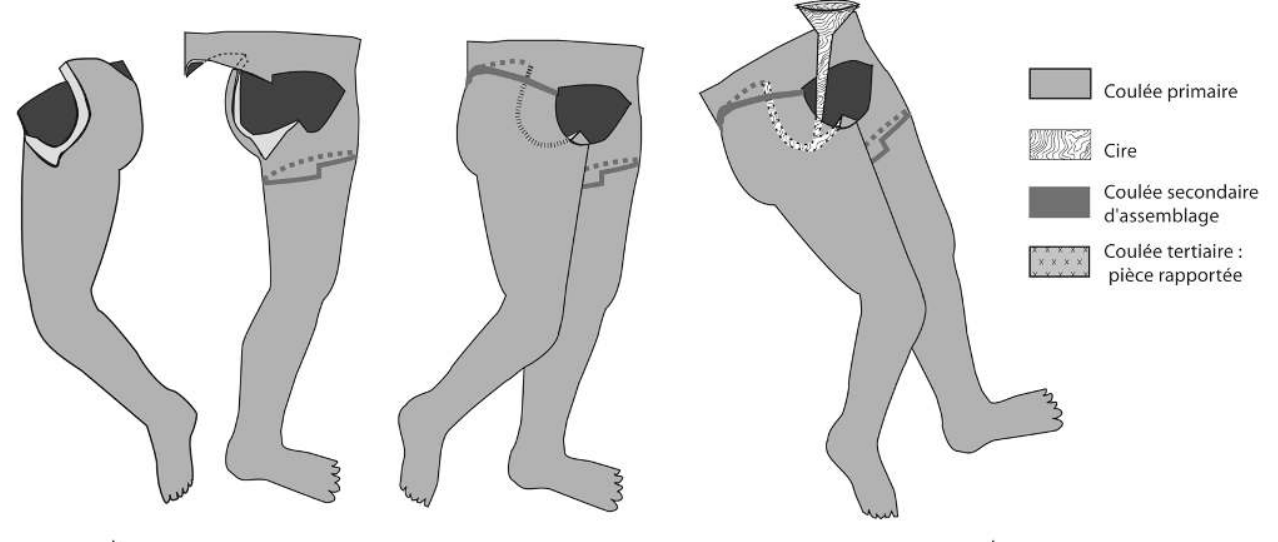

1

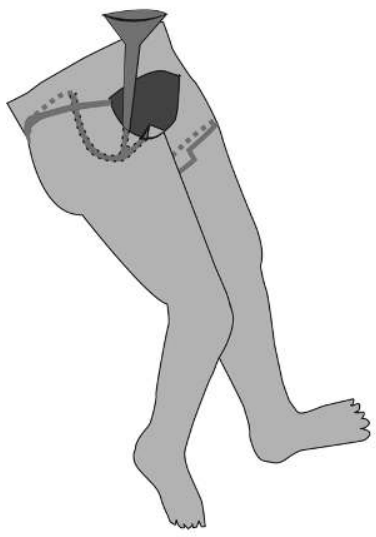

2

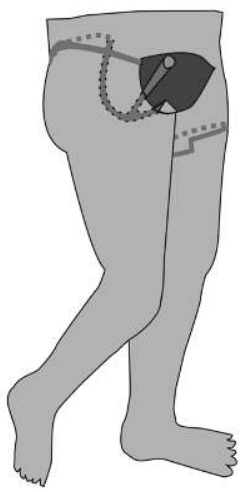

3

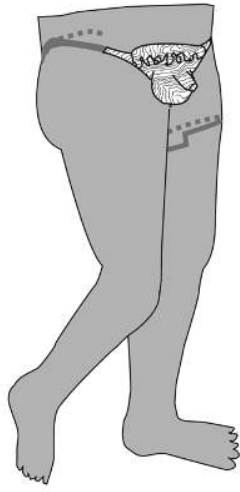

4

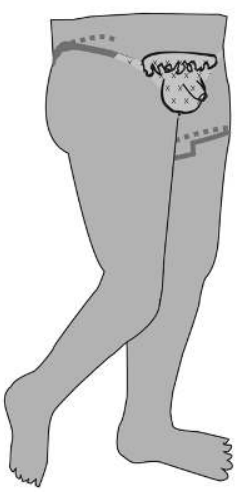

5
Figure 4: Statue de Jupiter, assemblage des jambes 1 . Préparation du soudage : les deux pièces à souder sont mises en contact, un système d'alimentation constitué d'un canal et d'un entonnoir est construit en cire, 2. Le soudage est réalisé par une coulée secondaire de bronze, 3. La partie externe du système d'alimentation est coupée, 4 . le sexe est ajouté en cire, 5 . Il est converti en métal par une coulée tertiaire. NB : les réfractaires mis en place pour contenir les métaux des coulées secondaires n'ont pas été représentés.

Figure 4 : Jupiter statue, joining of the legs: 1. Welding setting up: the two pieces to be weld are put in touch, a sprueing system (channel and funnel) is formed in wax 2. The welding is achieved by a secondary casting of bronze, directly poured on the pieces to be joined 3. The external part of the sprueing system is removed, 4. and 5. The sex is formed in wax, and then cast in metal. NB: The refractory materials used to contain the secondary castings are not drawn. de possibles jonctions entre les différentes pièces de cire, qui ont été ensuite assemblées pour former chacune des pièces de la statue. En ce qui concerne les assemblages par soudage, des préparations différentes ont été mises en évidence. La soudure de la tête sur le corps, située au milieu du cou, part d'une cuvette (à droite) et semble se transformer en un cordon faisant le tour de la jonction. Les assemblages des bras, situés au-dessous des épaules, se basent clairement sur des préparations en cuvette dont les traces sont visibles directement sur la statue (figure 3). La jonction de la jambe gauche sur le corps est parfaitement détectable en radiographie : le cordon de soudure passe au niveau du creux de l'aine pour remonter sur la hanche et revenir vers l'entrejambe par le bas des reins (figure 2, A). Il existe notamment une surface de contact relativement large aménagée entre les deux jambes (figure 2, F). Les réparations sont très peu nombreuses et servent souvent à masquer les lacunes laissées par les emplacements d'armatures ou des clous distanciateurs; elles prennent la formes de petites plaquettes rectangulaires et ont vraisemblablement été directement coulées en place (cou- lées secondaires de réparation). Notons la présence d'importantes accumulations de plomb dans les pieds qui doivent correspondre à des résidus du soclage de la statue. Enfin, les mamelons sont incrustés de cuivre non allié.

\section{La statue de Jupiter}

La statue de Jupiter $(0,92 \mathrm{~m})$, datée de la $2^{\mathrm{e}}$ moitié du $\mathrm{I}^{\mathrm{er}}$ siècle après J.-C., est constituée de sept pièces assemblées par soudage (tête, corps, les deux bras, les deux jambes, et le sexe) obtenues par le procédé indirect de fonte en creux à la cire perdue. L'alliage employé pour la confection de la statue de Jupiter se distingue de celui de la statue d'Apollon non seulement par sa composition chimique (proportion des majeurs et cortège d'impuretés différents), mais aussi par un meilleur contrôle de l'homogénéité de l'alliage d'une pièce à l'autre. Les coulées primaires sont faites d'un bronze à très forte teneur en plomb (teneur moyenne $\mathrm{Sn}: 5,3 \sigma 0,3 \% \mathrm{~m}$, $\mathrm{Pb}: 20,8 \sigma 0,9 \% \mathrm{~m})$. De plus, la tête offre un remarquable témoignage de maîtrise de la fonte à la cire perdue de par 
l'absence de réparations malgré une chevelure complexe, dont les boucles sont complètement creuses. En revanche, nous avons relevé une grande quantité de plaquettes de réparure (coulée secondaire de réparation de composition : $\mathrm{Sn}$ : 6,6 \%m, $\mathrm{Pb}: 15,6 \% \mathrm{~m})$. De forme rectangulaire pour les plus petites, polygonales pour les plus grandes, elles sont essentiellement localisées au niveau du haut du dos et des jonctions entre les différentes pièces, dans le but de masquer les défauts de coulée ou de soudage. Quelques clous distanciateurs ( $2 \mathrm{~mm}$ de côté) sont observables mais leur détection est rendue difficile du fait de la présence de nombreuses accumulations de métal au sein de la statue. Les assemblages par soudage (teneur moyenne Sn : 4,1 $\sigma 0,3 \%$ m, $\mathrm{Pb}: 17,7 \sigma 0,1 \% \mathrm{~m})$, se présentent sous plusieurs formes comme dans le cas d'Apollon. En premier lieu, deux de ces assemblages correspondent à des zones dont la lecture est difficile en raison de dégradations possiblement contemporaines de la fermeture du sanctuaire. Le fait que ces zones se soient révélées plus fragiles signifie vraisemblablement que les deux soudures comptaient de nombreux défauts. Depuis sa découverte, la statue a ainsi connu plusieurs campagnes de restauration relativement lourdes (Bonnin en 1845, la Maison André en 1960, le C2RMF en 2007) lors desquelles le bras gauche, retrouvé séparément du corps, a été remonté (plaques de métal et vis observables à la radiographie) et la déchirure à l'arrière de la cuisse gauche comblée et consolidée (ensemble de plaques de cuivre soudées à l'étain à l'intérieur et à l'extérieur de la statue). C'est pourquoi, hors leur localisation précise, il est difficile de réaliser une description détaillée de ces deux soudures. Néanmoins, on peut se rendre compte sur les radiographies, que comme pour le bras droit, le joint du bras gauche devait correspondre à une soudure en cordon. Par ailleurs, il est intéressant de remarquer le découpage non linéaire de la jonction entre le corps et la jambe gauche : ce découpage en " escalier" au niveau de la mi-cuisse facilitait certainement le blocage mécanique en prévision de l'assemblage par soudage. La jonction de la tête a quant à elle été effectuée par une succession de cuvettes formant un collier autour de la base du cou (figure 2, B). La zone de soudure de la jambe droite sur le corps semble suivre un découpage similaire à celui de la statue d'Apollon (figure 4). Cependant, son tracé est lisible en raison de la présence de nombreuses infiltrations de métal masquant certaines informations. Le cordon de soudure est supposé remonter au niveau du creux de l'aine - on y remarquera d'ailleurs la présence d'une grande plaquette de réparure polygonale (de l'ordre de 6 à $8 \mathrm{~cm}$ dans sa plus grande dimension) qui cache vraisemblablement des défauts de soudure - pour remonter au niveau de la hanche et revenir horizontalement vers l'entre-jambe. Mais surtout, l'examen radiographique de la zone de jonction a permis de mettre en évidence, à l'intérieur de la statue, un canal d'alimentation aujourd'hui en métal et à l'origine en cire, allant de l'ouverture du pubis (le sexe n'étant pas encore réalisé) vers l'entre-jambe, et dont la fonction probable était d'acheminer le bronze nécessaire à l'assemblage par soudage de la jambe droite sur le corps (figure 2, C). A la suite de quoi, la partie du système d'alimentation dépassant à l'extérieur de la statue a été coupée avant l'ajout du sexe par coulée secondaire (figure 3). Remarquons enfin la préservation exceptionnelle d'incrustations de cuivre au niveau des mamelons et des lèvres, et d'incrustations d'argent pour le blanc des yeux.

\section{Les fragments de bronze}

Les fragments découverts au XIX ${ }^{e}$ siècle ainsi que ceux issus de fouilles récentes constituent un ensemble d'une centaine de fragments qui portent souvent des déchirures et des déformations, témoignant d'une destruction volontaire avant l'enfouissement, probablement liée au rite spécifique de fermeture du sanctuaire. A l'exception de quatre bras provenant de statues de taille modeste (inférieure ou égale aux statues d'Apollon et de Jupiter), il est difficile d'identifier précisément l'iconographie de la plupart des pièces de ce dépôt; trois remontages seulement ont été retrouvés. Ces fragments semblent provenir d'une ou plusieurs statues de dimensions conséquentes, probablement monumentale(s). Une quarantaine de fragments représentatifs du lot ont été sélectionnés pour l'étude approfondie en laboratoire. Comme pour les statues de Jupiter et d'Apollon, des relevés technologiques et des prélèvements ont été réalisés sur chacun; les relevés ont été effectués à l'aide d'examens visuels et radiographiques. Deux exemples sont présentés en figure 2 (E, F) et figure 5, les résultats des analyses de la composition chimique des alliages étant rassemblés dans le tableau en annexe. Les informations technologiques recueillies ont été utilisées comme de véritables marqueurs et de nombreux rapprochements entre fragments ont de cette façon été mis en évidence. Ainsi, des emplacements de clous distanciateurs de $2 \mathrm{~mm}$ de côté sont détectables sur une dizaine de pièces. D'autres lacunes, correspondant à des traces laissées par des armatures circulaires ( $5 \mathrm{~mm}$ de diamètre) et/ou carrées ( $8 \mathrm{~mm}$ de coté), sont présentes sur plusieurs fragments. Elles sont souvent masquées par des plaquettes de réparure dont la fixation est renforcée par une brasure au plomb au revers, effectuée depuis l'intérieur de la statue dans les emplacements laissés vides à la suite du retrait des armatures. D'autres plaquettes de réparure de taille et de forme variables (de quelques $\mathrm{mm}^{2}$ à quelques $\mathrm{cm}^{2}$, rectangulaires 
ou polygonales) sont aussi utilisées pour masquer des défauts de fonderie (coulée primaire) ou de soudure (coulée secondaire). Les épaisseurs de paroi des coulées primaires sont en moyenne de 3,2 $\sigma 1,4 \mathrm{~mm}$. Comme pour les statues d'Apollon et de Jupiter, des assemblages par soudage sont observables sur de nombreux fragments. Cependant, la très grande majorité de ces cuvettes sont de forme quadrangulaire. Par exemple, le fragment issu de fouilles récentes (fig. 5) résulte de l'assemblage de deux pièces. Le joint semble être constitué d'une succession de cuvettes se chevauchant partiellement. L'autre fragment présenté (figure 5, fragment fouilles anciennes), également réalisé en deux parties, possède une cuvette de soudure sur une moitié de la jonction tandis que l'autre moitié, non soudée, est masquée par une plaquette de réparure. On notera par ailleurs la présence de nombreux épanchements de métal de soudure sur la surface interne des fragments, qui sont probablement la conséquence d'un travail peu soigné lors du processus d'assemblage. Des épanchements de plomb, situés au niveau des emplacements des plaquettes de réparure, sont également fréquents et correspondraient, comme pour les emplacements d'armatures, à un renfort de fixation des réparations par brasage.

Concernant les alliages utilisés, 34 coulées primaires sur 53 analysées possèdent entre 15 et $25 \% \mathrm{~m}$ en plomb et entre 6 et $10 \% \mathrm{~m}$ en étain, ce groupe est majoritairement constitué de fragments d'une ou plusieurs statues monumentales, sur lequel nous allons revenir de façon plus approfondie (cf. $\$$ nombre minimal de statues). D'autres pièces en revanche peuvent être considérées comme seule représentante de l'objet dont elles proviennent de par leur composition en éléments majeurs. C'est le cas des fragments de statuettes (échelle 1/4) tels que le bras décoré $(1,1 \% \mathrm{~m}$ en $\mathrm{Pb}$ et $12,3 \% \mathrm{~m}$ en $\mathrm{Sn}$, C2RMF 69343), le bras cuirassé (7,7 \%m en Pb et 8,2\%m en Sn, FZ 1515) (Gury et Guyard, 2006) et le fragment de pied (15,5\%m en $\mathrm{Pb}$ et $12 \% \mathrm{~m}$ en Sn, C2RMF 69507) mais aussi celui du bras plié $(3,7 \% \mathrm{~m}$ en $\mathrm{Pb}$ et $24,2 \% \mathrm{~m}$ en Sn, FZ 1517), qui est à associer à une statue de taille moyenne (échelle $1 / 2$ ). Un fragment de drapé qui appartenait à un grand bronze ne s'associe à aucune autre statue (2,3\%m en $\mathrm{Pb}$ et $4,5 \% \mathrm{~m}$ en $\mathrm{Sn}, \mathrm{C} 2 \mathrm{RMF}$ 69365). Les données issues de la composition des coulées secondaires d'assemblage et de réparation montrent que les fondeurs antiques ont eu recours à un métal d'apport de composition en éléments majeurs similaire à celui des coulées primaires, sauf dans le cas du fragment C2RMF 69373 (coulée primaire 10,2 \% m en $\mathrm{Pb}$ et $12,4 \% \mathrm{~m}$ en $\mathrm{Sn} /$ coulée secondaire de réparation 4,3\% en $\mathrm{Pb}$ et $4 \% \mathrm{~m}$ en $\mathrm{Sn}$ ) que l'on peut également considérer séparément du reste du dépôt.

\section{Nombre minimal de statues}

Les résultats précédents ont déjà permis de fournir non seulement une description de chacune des pièces du dépôt mais aussi de mettre en évidence les différences existant entre certains objets, ou lots de fragments : le fragment de plaque gravée et sa corniche, Jupiter, Apollon, les trois bras de statuettes (le bras décoré, le bras cuirassé et le bras tendu, échelle 1/4), le pied (échelle 1/4), le bras de statue (échelle $1 / 2$ ) et les deux fragments attribués à de la grande statuaire (C2RMF 69365 et C2RMF 69373). Cela nous permet d'ores et déjà d'affirmer l'existence de six statues, d'une base gravée, et de quatre statuettes indépendantes. Il reste maintenant à discuter le cas du groupe de fragments correspondant à une ou plusieurs statues monumentales. Nous avons remarqué de fortes similitudes lors des relevés technologiques et des analyses élémentaires de la composition des alliages sur une grande partie de ces fragments. Correspondent-ils à une seule et même statue? Afin de prendre en compte de manière simultanée l'ensemble des données fournies par notre étude, une analyse statistique exploratoire multivariée a été entreprise sur les concentrations élémentaires des coulées primaires (les éléments pris en compte sont: Ag, As, Au, Co, Fe, Ni, S, Zn, Pb, Sb, Sn) dans le but de vérifier plus précisément l'existence de liens entre ces fragments. Dans un premier temps, une classification ascendante hiérarchique (CAH) sur les données centréesréduites a permis de tester la similarité de ces pièces (mesure de la distance séparant chaque fragment dans la $\mathrm{CAH}$ ). À titre de comparaison, les données concernant la statue de Jupiter ont été incluses au sein de l'analyse statistique afin de fournir une référence pour la détermination du nombre de groupement statuaire (Jupiter devant nécessairement en former un). L'hypothèse de l'existence de cinq groupes au maximum a alors été proposée, à partir de laquelle un algorithme de K-moyennes (nuées dynamiques) a été appliqué. Cinq groupes aussi différents que possible les uns des autres ont été générés en maximisant la variance inter-classe et en minimisant la variance intra-classe. La figure 6 présente les groupes ainsi constitués, auxquels ont été ajoutés des données issues des relevés technologiques (clous distanciateurs et armatures, épaisseur des parois). Le groupe 5 correspond aux prélèvements effectués sur Jupiter (cf. paragraphe $L a$ statue de Jupiter). Le groupe 4 est constitué de 14 fragments, dont certains possèdent deux prélèvements qui correspondent à chaque pièce les constituant (G4 : teneur moyenne Sn : 9,2 $\sigma 1,1 \% \mathrm{~m}, \mathrm{~Pb}: 20,5 \sigma 2,4 \% \mathrm{~m})$ et rassemble en particulier toutes les pièces sur lesquelles on a pu observer des emplacements d'armatures carrées de $8 \mathrm{~mm}$ de côté. Par ailleurs, compte tenu de la présence des marqueurs tech- 


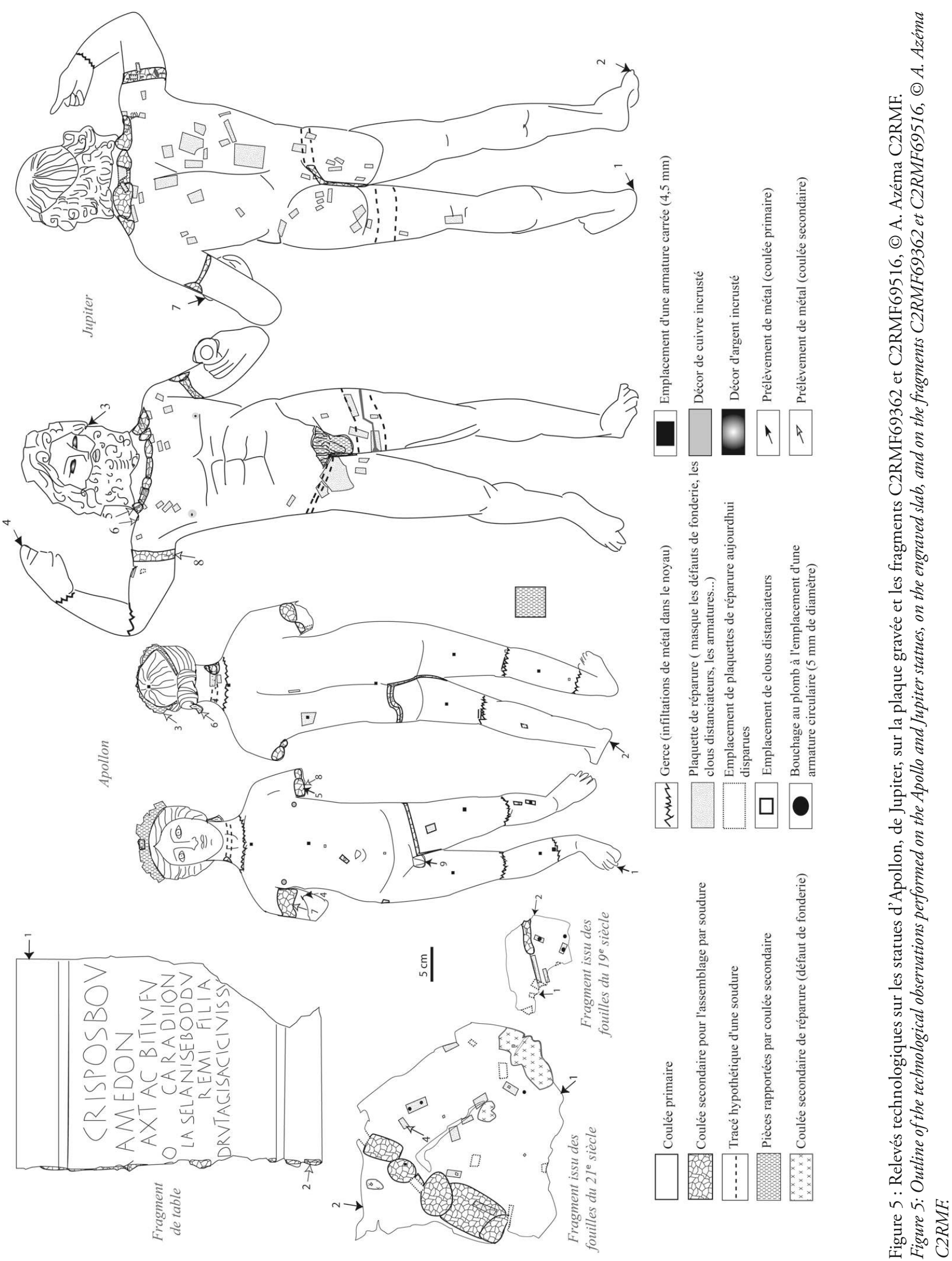




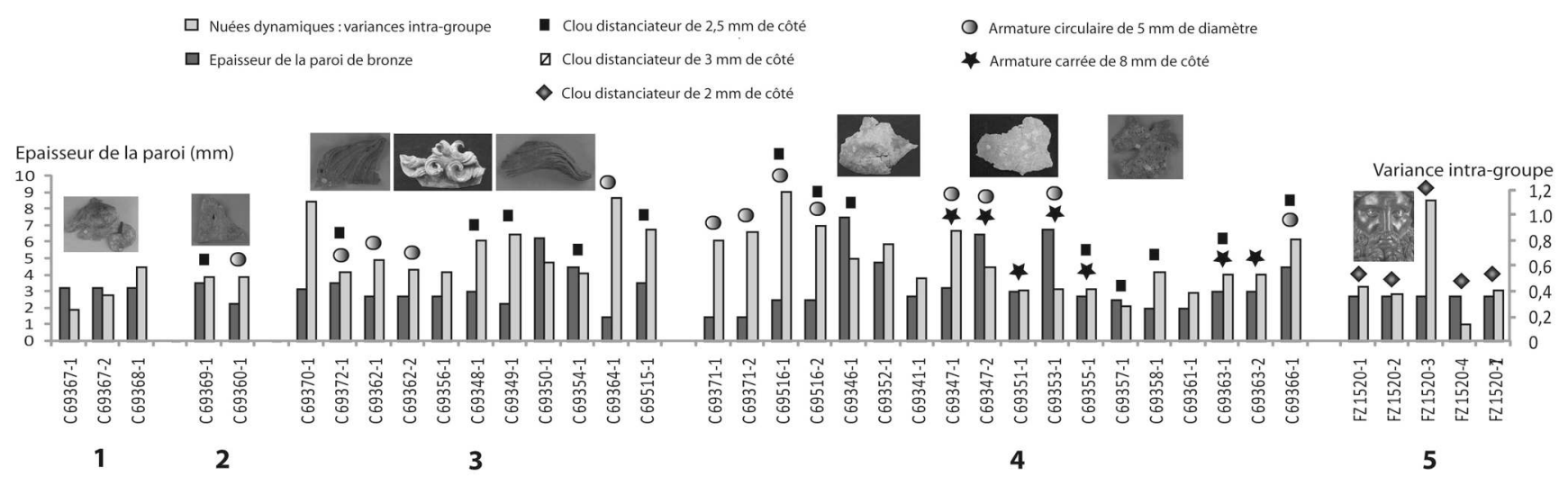

Figure 6 : Confrontation des résultats de l'étude statistique (nuées dynamiques) des compositions élémentaires des coulées primaires obtenues par le CEA Le Ripault avec les marqueurs technologiques les plus discriminants, (C) A. Azéma C2RMF.

Figure 6: Comparing the results of a statistical analysis ( $k$-means) on the elementary composition of the primary castings with the most discriminating technological observations, (C) A. Azéma C2RMF.

nologiques identiques (armatures circulaires de $5 \mathrm{~mm}$ de diamètre et clous distanciateurs de $2,5 \mathrm{~mm}$ de côté) on en déduit que les groupes 4 (G4), 3 (G3: 10 fragments, teneur moyenne $\mathrm{Sn}: 7,9 \sigma 1,2 \% \mathrm{~m}, \mathrm{~Pb}: 24,2 \sigma 3,9 \% \mathrm{~m})$ et 2 (G2: 2 fragments, teneur moyenne $\mathrm{Sn}: 8 \sigma 0,06 \% \mathrm{~m}, \mathrm{~Pb}$ : 20,7 $\sigma 0,05 \% \mathrm{~m})$ sont issus d'un même ensemble. Du seul point de vue de la composition des coulées primaires, les différences entre les groupes G2, G3 et G4 sont en réalité minimes et résident principalement dans les teneurs en zinc, fer, soufre. Ces impuretés sont particulièrement sensibles à l'oxydation, et les écarts peuvent être expliqués par des pertes qui auraient lieu lors de refontes répétées d'un même métal au sein de l'atelier. Le groupe 1, constitué de deux pièces formant un des remontages identifiés, se différencie clairement des autres par les teneurs en éléments majeurs de l'alliage employé (G1 : teneur moyenne $\mathrm{Sn}$ : 4,9 $\sigma$ 0,8 \%m, $\mathrm{Pb}: 10,4 \sigma 1,7 \% \mathrm{~m})$. Pourtant, le spectre d'impureté reste identique à celui des autres groupes de fragments, si on ne prend en compte que les éléments restant stables lors des refontes ( $\mathrm{Sb}, \mathrm{As}, \mathrm{Ag}, \mathrm{Au}$ ) (figure 7). Les fortes variations en éléments majeurs vis-à-vis des trois autres groupes ne signifient pas forcément que tous ces fragments ne font pas partie d'une même statue mais peuvent témoigner d'un choix volontaire de la part du fondeur, dépendant de la nature de la pièce et donc de son emplacement sur la statue. En effet, si l'on compare les aspects des fragments constituant chacune des classes, on remarque que G3 regroupe majoritairement des pièces à parois fines et à figuration de poils. G4, quant à lui, est constitué de pièces souvent lisses et à emplacement d'armatures carrées, qui sont aussi les plus épaisses. Dans le cas de G2, un des deux fragments peut être identifié comme le bas d'un vêtement. Enfin, si l'on revient au cas de G1, on reconnait clairement les testicules d'un animal pouvant être un cheval. On connaît plusieurs exemples de chevaux et de statues équestres monumentales où les organes génitaux formaient une pièce à part entière avant d'être assemblés sur le reste du corps : le Cheval du Musée du Capitole (Perrella, 2007), la statue équestre de Marc-Aurèle du Capitole (Formigli, 1999), le groupement équestre monumental de Cartoceto (G.M. Marini, 1987), les fragments des deux chevaux d'Augst (Janietz Schwarz \& Rouiller, 1996), ou encore la statue équestre récemment découverte lors des fouilles du Parc-Saint-Georges à Lyon (Darblade-Audoin et Mille, à paraitre). Il semble donc que la très grande majorité des fragments du dépôt de bronze, issus des fouilles anciennes mais aussi récentes, provient d'une seule et même statue monumentale, probablement équestre.

Finalement, en prenant en compte l'ensemble du dépôt, nous pouvons désormais affirmer qu'il est composé d'une base gravée (plaque et sa corniche), de trois moyens-bronzes (Jupiter, Apollon et la statue dont est issu le bras plié), de quatre statuettes (échelle 1/4) représentées par trois bras (le bras décoré, le bras cuirassé et le bras tendu) et un pied, mais aussi de deux grandes statues (au minimum à l'échelle un) dont il ne reste pour chacune qu'un fragment, et enfin d'une statue monumentale probablement équestre.

\section{Conclusion}

L'étude du dépôt de bronze découvert sur le site galloromain du Vieil-Evreux a permis de montrer la richesse de son contenu non seulement par la présence d'un nombre conséquent d'objets (onze statues et grandes statuettes, une base), en mettant par la même occasion en évidence une 
Figure 7 : Statistiques descriptives sur les groupes formés lors de l'application de l'algorithme des nuées dynamiques sur les compositions des coulées primaires, (C) A. Azéma C2RMF.

Figure 7 : Descriptive statistics for each of the groups obtained from the k-means clustering algorithm on the primary castings (C) A. Azéma C2RMF.
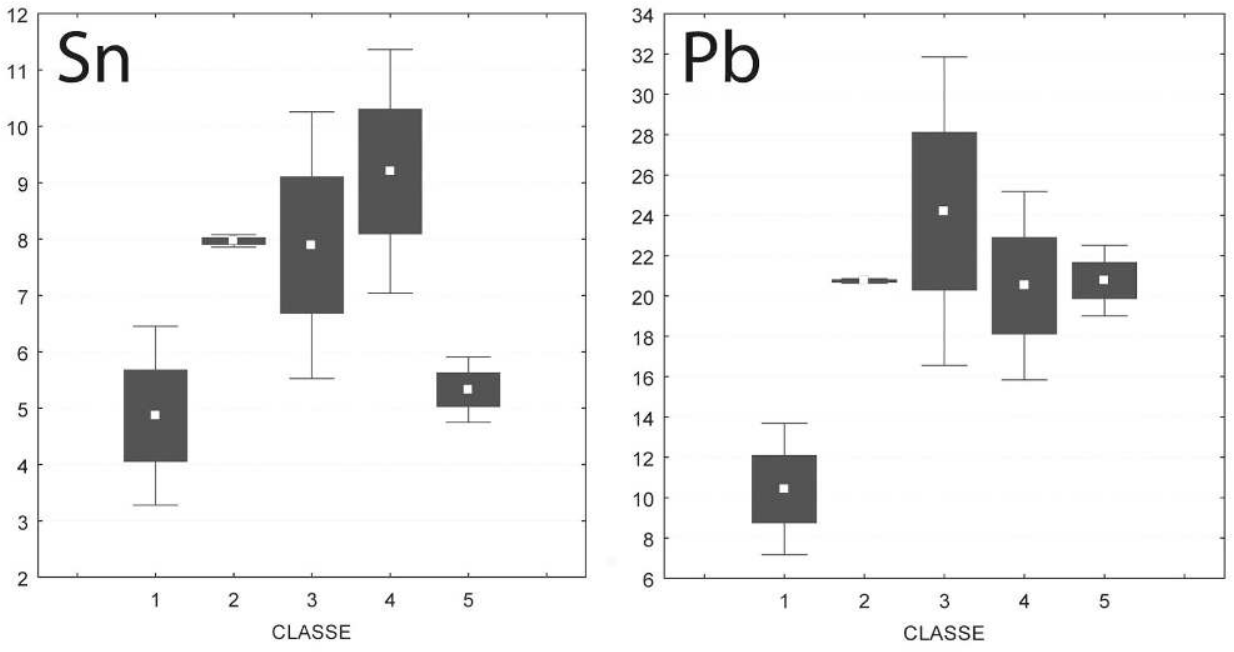

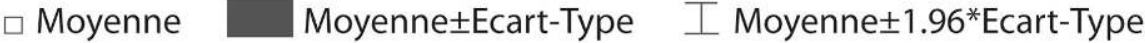
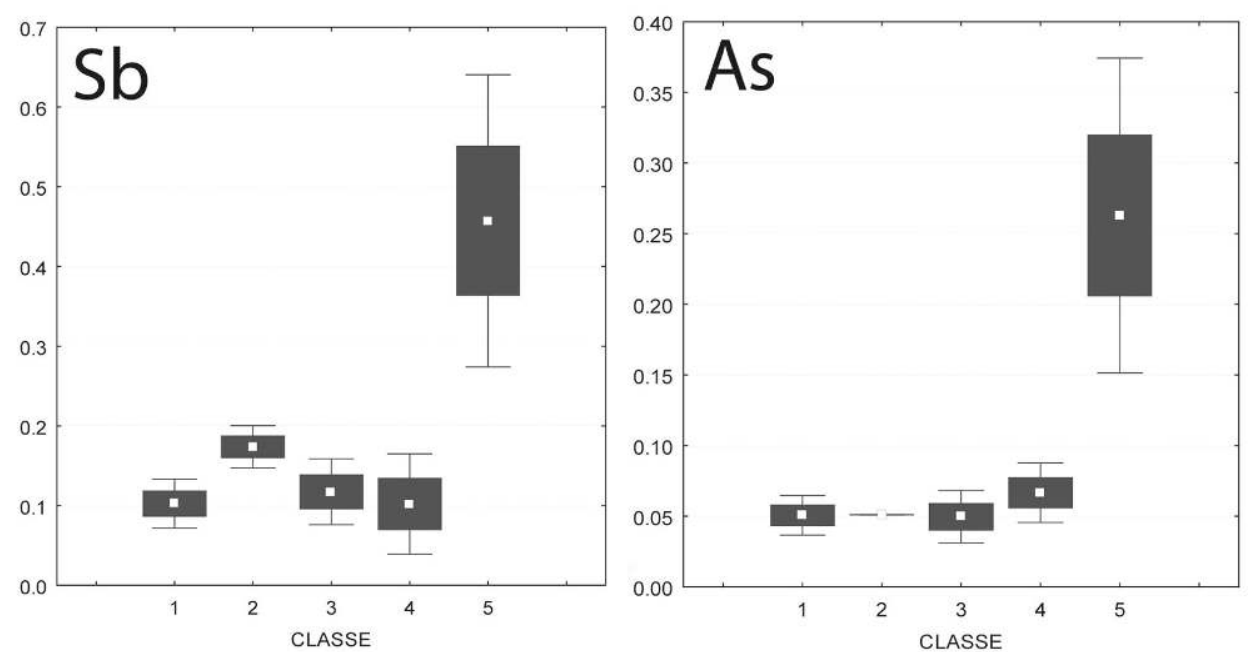

très grande maîtrise des techniques de fabrication de la statuaire métallique (fonte en creux à la cire perdue, soudage par fusion au bronze liquide...). L'élaboration d'une grande statue équestre par exemple, nécessite la mise en œuvre de procédés complexes et constitue un important chantier à l'échelle du sanctuaire du Vieil-Evreux. L'exceptionnelle facture de la statue de Jupiter en fait une des statues galloromaines les plus remarquables que nous connaissons aujourd'hui en France.

\section{Remerciements}

L'ensemble de ce travail n'aurait pu être réalisé sans le concours de nombreuses personnes que nous soubaitons remercier comme suit. Dans un premier temps, nous remercions le Musée d'Evreux en les personnes de L. Lecieux, C. Pannevel et $N$. Denninger de nous avoir permis de travailler sur ces remarquables objets. Merci à $S$. Cormier et $S$. Bertaudière de la MADE pour l'aide qu'ils nous ont apportée, en particulier lors de l'étude préliminaire de l'ensemble des fragments de bronze. Un grand merci à T. Borel (C2RMF) pour sa capacité exceptionnelle à faire apparaître les soudures et autres détails techniques sur ses radiographies. Merci également à D. Bagault (C2RMF) pour les photos d'ensemble mais aussi de détail qui ont été très utiles à la description des objets. Merci aux restaurateurs du C2RMF, D. Robcis, M. Leroux et C. Pariselle pour nos fructueux échanges et l'aide apportée. Enfin, nous remercions M. Szewczyk et C. Fontaine pour leur recherche et synthèse de données d'archives qui ont été indispensables à la relecture $d u$ dépôt de bronze du Vieil-Evreux. 


\section{Bibliographie}

AzÉma, A. et Mille, B., 2010 - La grande sculpture antique en bronze assemblée par soudage par fusion. Soudage et techniques connexes, Mars-Avril, Institut de Soudure, p. 20-22.

Azéma, A., Mille, B., Echégut, P. et De Sousa Meneses, D., 2012 - An experimental study of the welding techniques used on the Greek and Roman large Bronze Statues. The journal of the Historical Metallurgy Society 2010.

AzÉma, A., Mille, B., à paraître - The flow fusion welding process : a study of the joining techniques used on the Greek and Roman Large Bronze Statues. Proceeding Archaeometallurgy in Europe, 29 juin-1 july 2011, Bochum.

Bouquillon, A., Descamps, S., Hermary, A., Mille, B., 2006 - Une nouvelle étude de l'apollon Chatsworth, Revue Archéologique, 42, p. 227-261.

Bourgarit, D., Mille, B., 2003 - The elemental analysis of ancient copper-based artefacts by Inductively-CoupledPlasma Atomic-Emission-Spectrometry (ICP-AES) : an optimized methodology reveals some secrets of the Vix Crater. Measurement Science and Technology, 14, p. 1538-1555.

Darblade-Audoin, M-P., Mille, B., 2008 - Le pied de bronze colossal de Clermont-Ferrand, Monuments et mémoires de la fondation Eugène Piot, tome 87, Académie des Inscriptions et Belles-Lettres (Paris), p. 31-68.

Darblade-Audoin, M.-P., et Mille B., avec la collaboration de Pliska, P., à paraître - Présentation préliminaire à l'étude de la statue équestre du Parc-Saint-Georges à Lyon, dans Ayala, G. (ed.), les fouilles du Parc-Saint-Georges à Lyon, Documents d'Archéologie Française.

Formigli, E., 1999 - I Grandi Bronzi Antichi. Nuova Immagine. Italie.

Gury, F., Guyard, L., 2003 - Le sanctuaire central du Vieil-Evreux (Eure) et le bronze à l'épaule cuirassée, dans BrouquierReddé et al., Mars en Occident. Actes du colloque internatio- nal «Autours d'Allonnes (Sarthe), les sanctuaires de Mars en Occident ». Le Mans, université du Maine, 4-6 juin, PUR, p. 211-221.

Guyard, L., Bertaudière, S., 2007 - Le grand sanctuaire central du Vieil-Evreux (Eure) : Résultats de la campagne 2007. Haute-Normandie archéologie. Centre de recherches archéologiques de Haute Normandie, Rouen, Bulletin n ${ }^{\circ} 12$, p. 71-73.

Janietz Schwarz, B., Rouiller, D., 1996 - Ein Depot zerschlagener Grossbronzen aus Augusta Raurica : Die Rekonstruktion der beiden Pferdestatuen und Untersuchungen zur Herstellungstechnik, Forschungen in Augst, volume 20, 280 p.

Marini, G.M., 1987 - La teniche di fabbricazione, In Bronzi dorati da Cartoceto, Florence, p. 126-129.

Mille, B., 2007 - Etude technique du cheval de bronze de Neuvyen-Sullias, dans Gorget, C. et Guillaumet, J.-P., Le cheval et la danseuse, à la redécouverte du trésor de Neuvy-en-Sullias, Paris, Somogy, p. 88-99 et 264-265.

Mille, B. et Borel, T. 2007 - Composition élémentaire et radiographie des statuettes de Neuvy-en-Sullias, dans Gorget, C. et Guillaumet, J.-P. (eds.) Le cheval et la danseuse, à la redécouverte du trésor de Neuvy-en-Sullias, Paris, Somogy, p. 194-199 et 268-269.

Pariselle, C., 2011 - Rapport de restauration, Fragment de table inscrite inv. 4891, fragment de corniche inv. 4890, pour le musée municipal d'Evreux.

Perrella, C., Deloga, M., Parisi Presicce, C., 2007 - Il cavallo di bronzo nei Musei Capitolini : un original greco-roma. Punctum, Italie. 


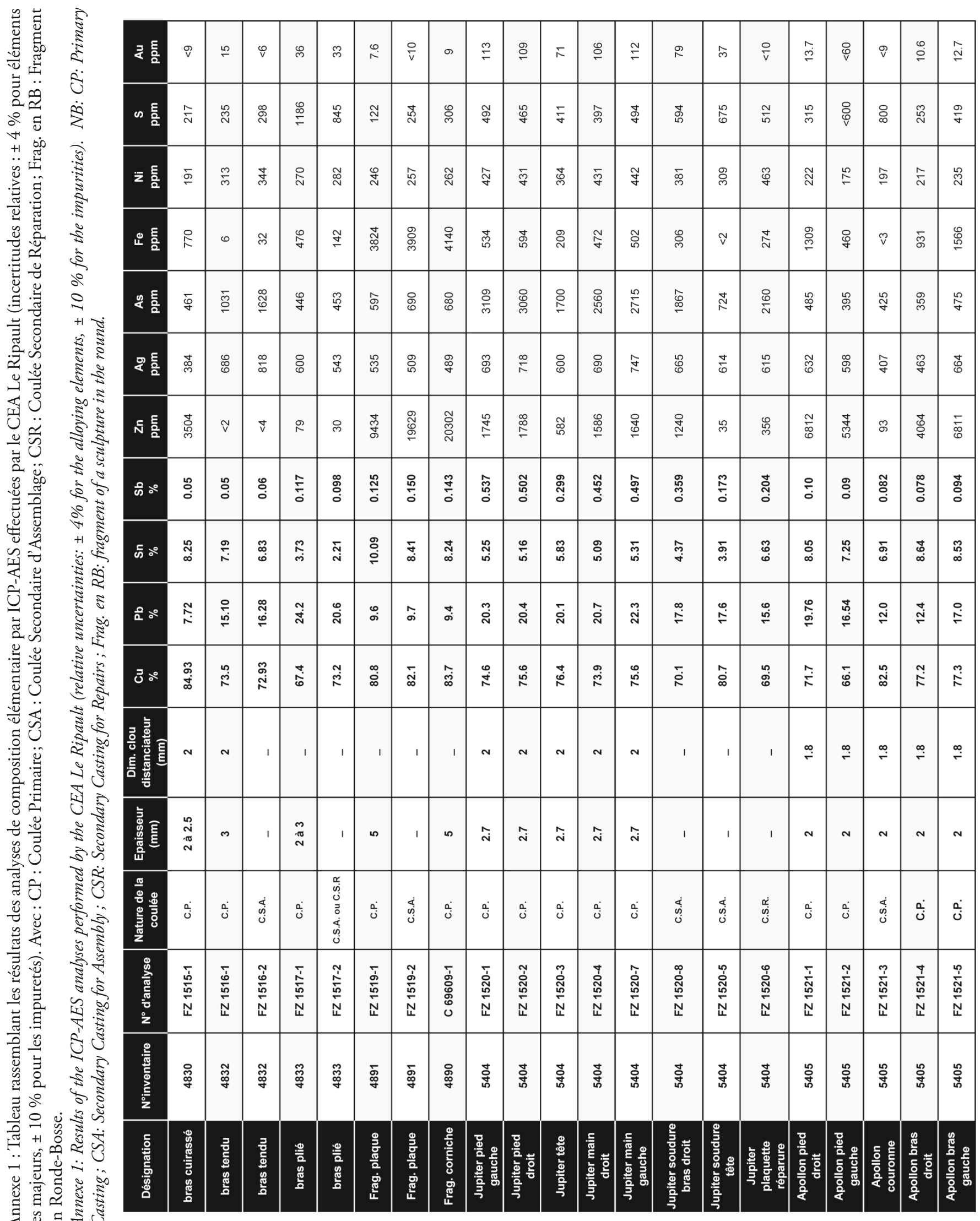




\begin{tabular}{|c|c|c|c|c|c|c|c|c|c|c|c|c|c|c|c|c|c|c|c|c|c|}
\hline$>\frac{E}{0}$ & v & $\bar{v}$ & $\bar{v}$ & $\bar{v}$ & $\bar{v}$ & $\bar{v}$ & $\bar{v}$ & $\bar{v}$ & $\bar{v}$ & $\bar{v}$ & 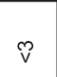 & $\bar{v}$ & $\bar{v}$ & $\bar{v}$ & $\bar{v}$ & 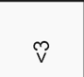 & $\bar{v}$ & $N$ & v & v & v \\
\hline$\supset \frac{\frac{g}{2}}{2}$ & $\underset{\text { \} }{\stackrel{\text { v }}{ }}} &{\stackrel{i}{v}} &{\frac{\stackrel{m}{g}}{v}} &{\underset{v}{R}} &{\stackrel{i}{v}} &{\text { Q }} &{\text { จे }} &{\text { ه্ }} &{\stackrel{?}{v}} &{\text { Dे }} &{\text { مْ }} &{\text { จे }} &{\stackrel{\mathscr{m}}{v}} &{\frac{\mathscr{m}}{v}} &{\text { จे }} &{\text { ه্ }} &{\stackrel{\mathscr{m}}{v}} &{\begin{array}{l}\frac{O}{0} \\
\frac{o}{v}\end{array}} &{\text { \& }} &{\underset{v}{\stackrel{i}{v}}} &{\text { : }} \\
{\hline \text { F }} &{\vartheta} &{\text { v }} &{\text { i }} &{\text { v }} &{\text { v }} &{\text { v }} &{\text { v }} &{\text { v }} &{\text { v }} &{\text { v }} &{\widehat{v}} &{\text { v }} &{\dot{v}} &{\dot{v}} &{\text { v }} &{\grave{v}} &{\text { ป }} &{\stackrel{\mathscr{p}}{v}} &{\vartheta} &{\vartheta} &{\vartheta} \\
{\hline \stackrel{\circ}{\vdash} \frac{\bar{z}}{2}} &{\hat{m}} &{\stackrel{m}{=}} &{\simeq} &{\frac{0}{v}} &{\frac{\varrho}{v}} &{\underset{\mathcal{N}}{\mathbb{N}}} &{\text { ํ }} &{\stackrel{\widetilde{v}}{v}} &{\text { i }} &{\text { ని }} &{\text { 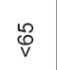 }} &{\stackrel{\nabla}{v}} &{\text { ठे }} &{\ddot{\vartheta}} &{\text { จ }} &{\mathscr{~}} &{\stackrel{2}{N}} &{\text { 品 }} &{\stackrel{\circ}{V}} &{\text { ه্ }} &{\text { ৪ }} \\
{\hline \text { œ }} &{\stackrel{\circ}{ }} &{\text { ஸ્ }} &{\stackrel{\circ}{\circ}} &{\frac{\infty}{v}} &{\frac{\infty}{v}} &{\stackrel{\sim}{v}} &{\text { 广 }} &{\stackrel{\mathscr{v}}{v}} &{\stackrel{i}{v}} &{\stackrel{\vec{v}}{v}} &{\stackrel{m}{\rightleftharpoons}} &{\text { \& }} &{8} &{\text { in }} &{\text { \& }} &{\stackrel{\circ}{ }} &{\text { 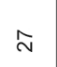 }} &{\stackrel{\text { S }}{\sim}} &{\tilde{\sigma}} &{\text { 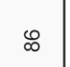 }} &{\stackrel{0}{1}} \\
{\hline \text { 을 }} &{\frac{8}{v}} &{\stackrel{\mathscr{v}}{\mathrm{v}}} &{\underset{v}{p}} &{\stackrel{\leftrightarrow}{\sim}} &{\mathscr{\mathscr { v }}} &{\text { 昌 }} &{\text { \& }} &{\stackrel{\text { \& }}{v}} &{\mathscr{p}} &{\stackrel{?}{v}} &{\stackrel{\stackrel{m}{v}}{\mathrm{~m}}} &{\stackrel{8}{v}} &{\underset{v}{q}} &{\underset{v}{\stackrel{R}{v}}} &{\text { సి }} &{\frac{m}{v}} &{\underset{v}{R}} &{\text { 呑 }} &{\frac{8}{v}} &{\frac{\circ}{v}} &{\frac{8}{v}} \\
{\hline \stackrel{\circ}{\frac{0}{\Sigma}} \frac{\bar{\partial}}{2}} &{\text { ర్ల }} &{\stackrel{\circ}{v}} &{\stackrel{\mathscr{R}}{v}} &{\stackrel{\circ}{v}} &{\stackrel{\circ}{v}} &{\frac{L}{v}} &{\stackrel{\circ}{v}} &{\frac{n}{v}} &{\frac{O}{v}} &{\frac{\omega}{v}} &{\text { 导 }} &{\frac{\varrho}{v}} &{\stackrel{\sim}{\sim}} &{\stackrel{\sim}{\sim}} &{\frac{\mathscr{L}}{v}} &{\text { \& }} &{\stackrel{\sim}{v}} &{\text { 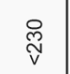 }} &{\text { ণ్ల }} &{\text { প్v }} &{\stackrel{\mathscr{\vartheta}}{v}} \\
{\hline} &{\text { N }} &{\text { : }} &{\stackrel{m}{\stackrel{m}{v}}} &{\hat{o}} &{=} &{\text { 官 }} &{\text { ָี }} &{\text { if }} &{\stackrel{\leftrightarrow}{\infty}} &{\text { 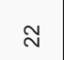 }} &{\underset{\infty}{\sim}} &{\stackrel{0}{\sigma}} &{\stackrel{\circ}{\dot{q}}} &{\stackrel{\mathcal{J}}{\mathcal{J}}} &{\Xi} &{\bar{v}} &{\stackrel{0}{\circ}} &{\dot{v}} &{\text { on }} &{\stackrel{m}{i}} &{\stackrel{m}{\dot{q}}} \\
{\hline \frac{\text { 일 }}{\Sigma}} &{\overline{0}} &{\overline{v_{v}^{\prime}}} &{\bar{\sigma}} &{\stackrel{m}{+}} &{\bar{q}} &{\bar{q}} &{\bar{q}} &{\bar{c}} &{\bar{j}} &{\text { मे }} &{\bar{v}} &{\bar{\sigma}} &{\bar{\sigma}} &{\overline{\bar{v}}} &{\stackrel{+}{\circ}} &{\tilde{N}} &{\overline{\bar{v}}} &{\stackrel{m}{p}_{i}^{p}} &{\bar{o}} &{\bar{o}_{\mathrm{j}}} &{\bar{c}} \\
{\hline \underline{\underline{5}}} &{\bar{N}} &{\stackrel{\text { จ }}{\mathrm{v}}} &{\text { g }} &{\text { 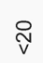 }} &{\stackrel{\nabla}{v}} &{\text { ণ }} &{\text { 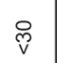 }} &{\text { ه্ণ }} &{\stackrel{\nabla}{v}} &{\text { סे }} &{\text { 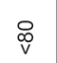 }} &{\text { ஜे }} &{\text { O }} &{\text { g }} &{\text { ర్ }} &{\text { 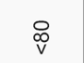 }} &{\text { g }} &{\text { 导 }} &{\stackrel{\circ}{\ominus}} &{8} &{\stackrel{\circ}{\circ}} \\
{\hline \text { 옾흠 }} &{\text { }} &{\stackrel{\circ}{v}} &{\stackrel{\infty}{V}} &{\stackrel{\circ}{v}} &{\stackrel{\circ}{v}} &{\stackrel{N}{V}} &{\stackrel{\tilde{V}}{v}} &{\stackrel{N}{v}} &{\stackrel{\circ}{v}} &{\stackrel{N}{v}} &{\begin{array}{l}\mathscr{D} \\
\stackrel{\nabla}{v}\end{array}} &{\stackrel{\tilde{v}}{v}} &{\stackrel{\infty}{v}} &{\stackrel{\infty}{v}} &{\stackrel{\tilde{v}}{v}} &{\text { 品 }} &{\stackrel{\infty}{v}} &{\frac{\infty}{\grave{v}}} &{\text { 宁 }} &{\text { ه్ల }} &{\text { O్ర }} \\
{\hline \text { \& } \frac{\bar{g}}{2}} &{\text { 宁 }} &{\stackrel{\circ}{v}} &{\stackrel{\vec{V}}{v}} &{\stackrel{\circ}{v}} &{\stackrel{\circ}{v}} &{\frac{\mathscr{L}}{V}} &{\stackrel{\circ}{v}} &{\frac{n}{v}} &{\stackrel{\circ}{v}} &{\frac{\omega}{v}} &{\text { P }} &{\frac{\infty}{v}} &{\underset{\mathcal{V}}{\text { vat }}} &{\stackrel{\sim}{v}} &{\frac{\mathscr{L}}{v}} &{\text { g }} &{\stackrel{\vec{v}}{v}} &{\text { 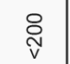 }} &{\text { Q్ల }} &{\text { ᄋ్ల }} &{\text { 䒽 }} \\
{\hline \text { ¿ }} &{\dot{v}} &{\checkmark} &{\vartheta} &{\text { 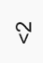 }} &{\checkmark} &{\checkmark} &{\text { v }} &{v} &{\text { v }} &{\sim} &{\stackrel{\vartheta}{v}} &{\text { 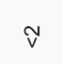 }} &{\phi} &{\vartheta} &{\checkmark} &{\vartheta} &{\vartheta} &{\hat{v}} &{\text { J }} &{\dot{v}} &{\dot{v}} \\
{\hline 8 \frac{5}{2}} &{\simeq} &{\text { 串 }} &{\underset{⿱ 亠}{\stackrel{\Gamma}{\Gamma}}} &{\stackrel{L}{\infty}_{\infty}^{\circ}} &{\stackrel{\circ}{-}} &{\stackrel{m}{\sim}} &{F} &{\simeq} &{\hat{\circ}} &{\text { F }} &{\text { 움 }} &{\infty} &{\text { मे }} &{\text { 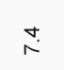 }} &{\text { 号 }} &{ \pm} &{\stackrel{\text { }}{\sim}} &{\text { }} &{\text { v }} &{\bar{\infty}} &{\stackrel{+}{\mathrm{N}}} \\
{\hline \text { 응 } \frac{\bar{z}}{2}} &{\vartheta} &{\bar{v}} &{\text { v }} &{\text { v }} &{\text { 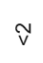 }} &{\text { v }} &{\text { v }} &{\text { v }} &{\bar{v}} &{\text { v }} &{\stackrel{\checkmark}{\mathrm{V}}} &{\sim} &{v} &{\text { v }} &{\text { v }} &{\dot{v}} &{\text { v }} &{\bar{v}} &{\vartheta} &{\tilde{v}} &{\text { จ }} \\
{\hline \overline{\mathbf{m}} \overline{\mathrm{g}}} &{\underset{\text { Nิ }}{\text { N }}} &{\text { 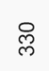 }} &{\text { 苛 }} &{\underset{\mathrm{v}}{\stackrel{8}{v}}} &{\underset{\substack{\infty \\
v}}{\infty}} &{\frac{8}{v}} &{\frac{8}{v}} &{\frac{8}{v}} &{\underset{\mathrm{v}}{\otimes}} &{\frac{o}{v}} &{\stackrel{\circ}{\stackrel{v}{v}}} &{\frac{o}{v}} &{\frac{O}{V}} &{\frac{\rho}{v}} &{\frac{8}{v}} &{\frac{O}{\mathrm{v}}} &{\frac{\stackrel{\circ}{V}}{\mathrm{v}}} &{\frac{O}{\frac{O}{V}}} &{\text { స్ ָ }} &{\frac{O}{v}} &{\text { స్్ }} \\
{\hline \text { 离 } \frac{\mathrm{g}}{2}} &{\stackrel{\circ}{\stackrel{\circ}{~}}} &{\text { 品 }} &{\text { : }} &{\text { : }} &{\text { б. }} &{\overline{0}} &{\text { @̊ }} &{\text { ঃே }} &{\text { tr }} &{\hat{o}} &{\check{F}} &{\stackrel{m}{0}} &{\stackrel{\circ}{\stackrel{\circ}{\circ}}} &{\stackrel{\mathscr{L}}{\infty}} &{\stackrel{\infty}{\infty}} &{\text { म্ল }} &{\text { 怘 }} &{\stackrel{\infty}{\check{\infty}^{\infty}}} &{\text { : }} &{\text { : }} &{\stackrel{0}{\circ}} \\
{\hline \text { ¿ } \frac{\bar{z}}{2}} &{\frac{n}{v}} &{\stackrel{\vartheta}{\vee}} &{\stackrel{\circ}{v}} &{\text { 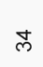 }} &{\stackrel{m}{\rightleftarrows}} &{\hat{v}} &{\stackrel{\circ}{v}} &{\text { o }} &{\vartheta} &{\text { 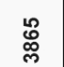 }} &{\stackrel{\vec{v}}{v}} &{\hat{v}} &{\frac{n}{v}} &{\stackrel{\circ}{v}} &{\hat{v}} &{\stackrel{\mathrm{v}}{\mathrm{v}}} &{\stackrel{m}{q}} &{\frac{8}{v}} &{\stackrel{\varrho}{v}} &{\frac{\infty}{v}} &{\frac{\infty}{v}} \\
{\hline \text { 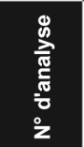 }} &{\frac{\bar{\sigma}}{\stackrel{5}{0}}} &{\begin{array}{l}\bar{D} \\
\stackrel{0}{0} \\
\stackrel{N}{N}\end{array}} &{\begin{array}{l}\multirow{2}{0}{} \\
\stackrel{5}{5} \\
\stackrel{N}{N}\end{array}} &{\text { ì }} &{\text { N⿳亠口冋. }} &{\bar{\delta}} &{\text { 崩 }} &{\text { 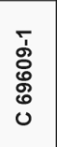 }} &{\text { }} &{\text { ָָ }} &{\text { 商 }} &{\text { 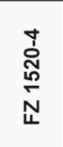 }} &{\text { 啇 }} &{\text { 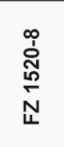 }} &{\text { 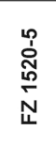 }} &{\begin{array}{l}0 \\
\stackrel{i}{N} \\
\stackrel{N}{N} \\
\mathbb{L}\end{array}} &{\begin{array}{l}\stackrel{T}{\grave{N}} \\
\stackrel{N}{N} \\
\stackrel{N}{L}\end{array}} &{\frac{\stackrel{N}{N ָ}}{\stackrel{N}{N}}} &{\begin{array}{l}\stackrel{m}{N} \\
\stackrel{N}{N} \\
\mathbb{L}\end{array}} &{\text { 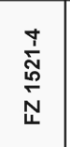 }} &{\frac{\text { }}{\frac{1}{\pi}}} \\
{\hline \text { 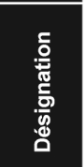 }} &{\text { 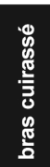 }} &{\text { 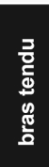 }} &{\text { 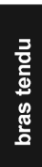 }} &{\text { 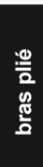 }} &{\begin{array}{l}\frac{0}{0} \\
\frac{0}{0} \\
\frac{0}{0}\end{array}} &{\begin{array}{l}\frac{9}{8} \\
\frac{0}{0} \\
\frac{0}{6}\end{array}} &{\text { 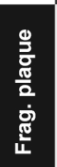 }} &{\text { 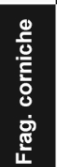 }} &{\text { 응 }} &{\text { 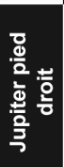 }} &{\text { 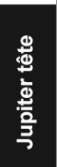 }} &{\text { 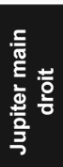 }} &{\text { 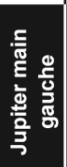 }} &{\text { 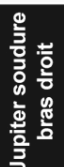 }} &{\text { 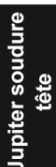 }} &{\text { 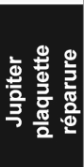 }} &{\text { 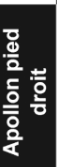 }} &{\text { 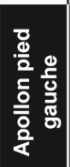 }} &{\text { 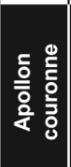 }} &{\text { 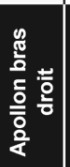 }} &{\text { 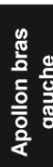 }} \\
$\hline
\end{tabular}




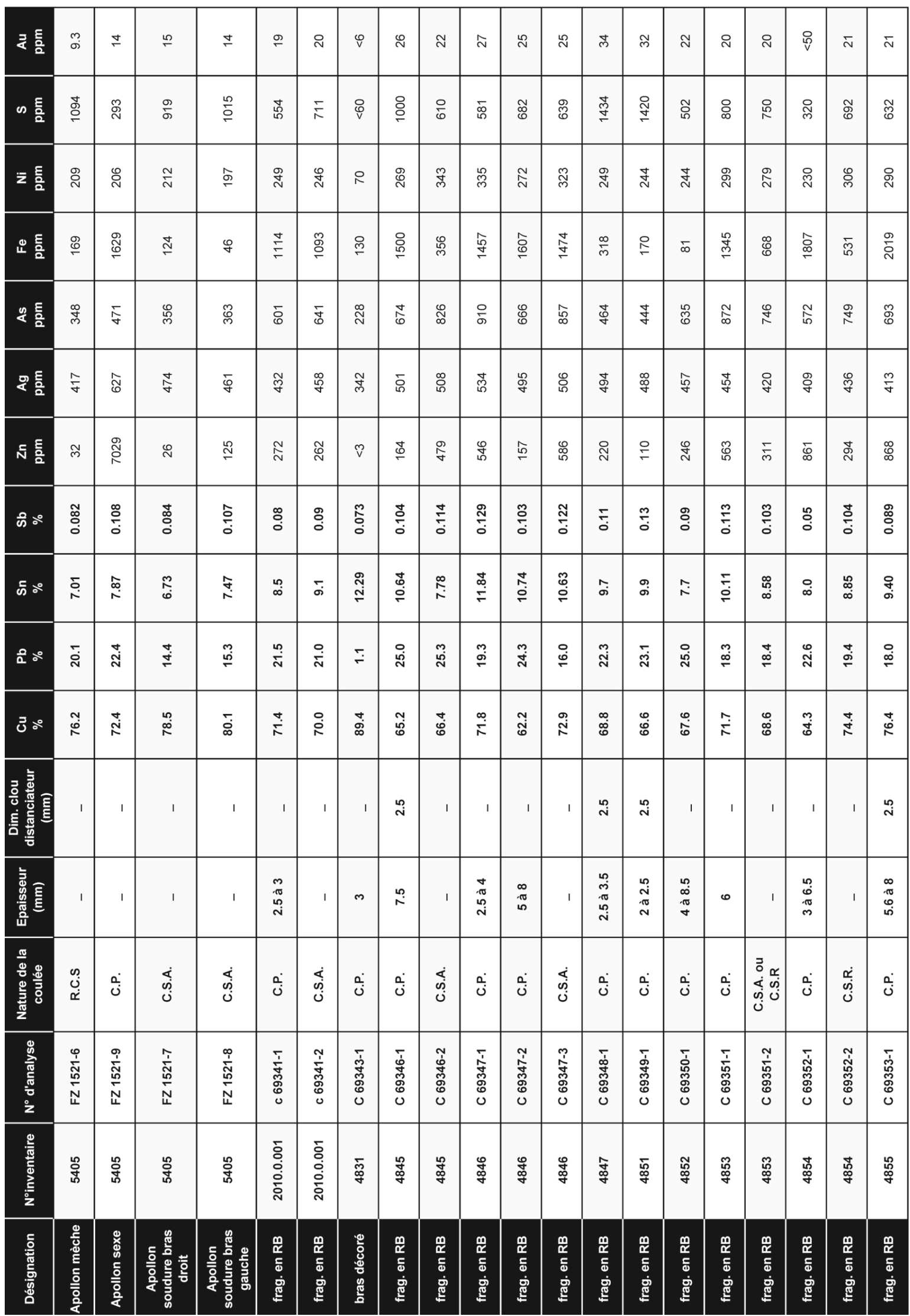




\begin{tabular}{|c|c|c|c|c|c|c|c|c|c|c|c|c|c|c|c|c|c|c|c|c|}
\hline$>\frac{5}{2}$ & $\bar{v}$ & v & $\stackrel{a}{i}$ & v & $\stackrel{\circ}{i}$ & $\stackrel{\leftrightarrow}{\dot{m}}$ & $\bar{v}$ & $\tilde{N}$ & $\bar{v}$ & v & $\tilde{v}$ & $\bar{v}$ & $\stackrel{\mathscr{N}}{\mathrm{i}}$ & $\stackrel{\sim}{\sim}$ & v & $\begin{array}{c}\infty \\
\vec{v}\end{array}$ & $\stackrel{\circ}{\circ}$ & 음 & ${ }_{\substack{\infty \\
v}}$ & $\begin{array}{l}\infty \\
\stackrel{0}{0}\end{array}$ \\
\hline$\supset \frac{\xi}{2}$ & $\frac{\mathscr{m}}{v}$ & ষ্ণ & 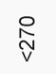 & 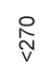 & 品 & 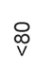 & $\frac{\mathscr{m}}{v}$ & $\stackrel{8}{\mathrm{~V}}$ & $\frac{\mathscr{m}}{v}$ & $\frac{\mathscr{O}}{\vec{v}}$ & 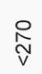 & $\frac{\mathscr{m}}{v}$ & $\stackrel{8}{\mathrm{v}}$ & $\stackrel{8}{\vee}$ & 品 & จ & $\underset{v}{\stackrel{P}{v}}$ & 号 & 암 & จ \\
\hline$F \bar{g}$ & $\dot{v}$ & $\stackrel{\vartheta}{v}$ & $\hat{v}$ & $\widehat{v}$ & $\stackrel{\circ}{\text { i }}$ & $\tilde{v}$ & $\dot{v}$ & v & $\dot{v}$ & $\stackrel{\vartheta}{v}$ & $\widehat{v}$ & $\dot{v}$ & $\stackrel{\text { ì }}{ }$ & $\stackrel{m}{\sim}$ & $\stackrel{\circ}{\leftarrow}$ & $\tilde{v}$ & v & $\bar{v}$ & v & v \\
\hline$\stackrel{\circ}{\circ}$ & $\stackrel{\phi}{\nabla}$ & ڤ̊ & 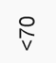 & $\stackrel{q}{v}$ & 郘 & 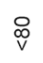 & প్ర & $\underset{\mathcal{N}}{\mathcal{N}}$ & ঠ్ & $\stackrel{g}{V}$ & $\underset{\mathrm{V}}{\mathrm{V}}$ & $\stackrel{\circ}{\circ}$ & 实 & ᄋ & 岀 & $\tilde{\widetilde{v}}$ & $\frac{O}{v}$ & 品 & $\tilde{\nu}$ & $\tilde{\widetilde{v}}$ \\
\hline$\stackrel{8}{\circ}$ & o & 郘 & $\underset{\substack{\mathrm{V} \\
\text { d. }}}{ }$ & 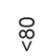 & O & 吕 & sิ & $\stackrel{\sim}{\mathrm{V}}$ & P & 郘 & 오v & o & 弚 & 官 & O্ত & g & $\frac{\infty}{v}$ & $\stackrel{\circ}{\nabla}$ & o & ơ \\
\hline a. $\frac{5}{2}$ & $\stackrel{q}{v}$ & $\frac{O}{v}$ & $\frac{g}{v}$ & $\frac{g}{v}$ & $\stackrel{\circ}{\mathrm{v}}$ & 品 & $\underset{v}{q}$ & 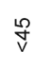 & $\underset{v}{\stackrel{q}{v}}$ & $\frac{8}{v}$ & 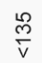 & $\underset{\substack{v \\
v}}{ }$ & $\stackrel{m}{m}$ & $\frac{n}{v}$ & O্ & $\stackrel{p}{v}$ & $\stackrel{\leftrightarrow}{\sigma}$ & סे & 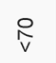 & $\stackrel{i}{i}$ \\
\hline$\stackrel{\circ}{\circ} \frac{\mathrm{g}}{2}$ & $\stackrel{\mathscr{N}}{\mathbf{V}}$ & ठ্ & 导 & 导 & $\hat{v}$ & $\stackrel{\circ}{v}$ & $\stackrel{\sim}{v}$ & $\stackrel{\circ}{v}$ & $\stackrel{\mathscr{V}}{v}$ & O & 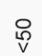 & $\stackrel{\circ}{\circ}$ & $\hat{v}$ & $\hat{v}$ & $\hat{v}$ & $\stackrel{\mathscr{v}}{v}$ & $\frac{0}{v}$ & $\hat{v}$ & $\stackrel{\sim}{v}$ & $\stackrel{\mathscr{N}}{\mathrm{v}}$ \\
\hline$\frac{c}{\frac{c}{2}} \frac{\bar{\partial}}{2}$ & $\stackrel{\vec{D}}{N}$ & $\stackrel{\circ}{\circ}$ & $\bar{v}$ & $\bar{v}$ & $\stackrel{\sim}{\sim}$ & v & $\stackrel{\infty}{\circ}$ & $\stackrel{\uparrow}{-}$ & ઈิ & 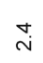 & $\stackrel{m}{\longrightarrow}$ & نొ & $\bar{v}$ & $\bar{v}$ & $\bar{v}$ & مُ & $\infty$ & $\stackrel{\sim}{\sim}$ & $\stackrel{\circ}{\circ}$ & $\hat{i}$ \\
\hline$\frac{\text { 일 }}{2}$ & $\bar{i}$ & $\bar{c}$ & $\bar{v}$ & $\bar{\sigma}$ & $\infty$ & 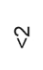 & $\bar{q}$ & $\bar{v}$ & $\bar{v}_{\bar{v}}$ & $\bar{v}$ & $\stackrel{10}{\circ}$ & $\bar{v}$ & $\stackrel{m}{N}$ & $\bar{v}$ & $\bar{v}$ & $\bar{i}$ & is & $\bar{v}$ & $\bar{q}_{\bar{v}}^{-}$ & $\bar{v}$ \\
\hline 도음 & g & $\stackrel{\circ}{\mathrm{V}}$ & 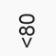 & $\underset{\mathscr{V}}{\stackrel{D}{ }}$ & 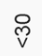 & 号 & g & $\stackrel{\mathscr{v}}{\tilde{v}}$ & g & $\stackrel{\circ}{\mathrm{V}}$ & $\underset{\substack{\infty \\
v}}{\infty}$ & g & $\stackrel{\vartheta}{v}$ & 官 & 宅 & g d & $\stackrel{\nabla}{v}$ & $\stackrel{\mathscr{v}}{v}$ & g & g v \\
\hline 옾흠 & $\frac{\infty}{V}$ & ర్ల & $\underset{\mathscr{v}}{\mathscr{v}}$ & $\ddot{\mathscr{v}}$ & $\stackrel{\phi}{\nabla}$ & $\stackrel{8}{v}$ & $\frac{\infty}{v}$ & $\frac{N}{v}$ & $\stackrel{\infty}{v}$ & $\underset{v}{\mathbb{V}}$ & $\ddot{\mathscr{v}}$ & $\stackrel{\infty}{v}$ & $\stackrel{\nabla}{\nabla}$ & $\stackrel{\nabla}{v}$ & 宅 & $\stackrel{\infty}{v}$ & $\frac{\circ}{\mathrm{v}}$ & $\ddot{v}$ & $\frac{\infty}{v}$ & $\stackrel{\infty}{V}$ \\
\hline \& $\frac{5}{2}$ & $\stackrel{乛}{v}$ & סิ & g v & g & $\frac{\circ}{v}$ & $\frac{\mathscr{L}}{v}$ & $\stackrel{\vec{v}}{v}$ & $\frac{m}{v}$ & $\stackrel{\vec{v}}{\mathrm{v}}$ & ס्ల & g & $\stackrel{\vec{V}}{V}$ & $\stackrel{\circ}{v}$ & $\stackrel{\circ}{v}$ & $\frac{\circ}{v}$ & $\stackrel{\vec{v}}{v}$ & $\stackrel{\circ}{\mathrm{V}}$ & $\stackrel{\circ}{\mathrm{V}}$ & 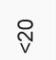 & $\stackrel{i}{v}$ \\
\hline ¿̇ & 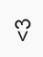 & $\dot{v}$ & $\vartheta$ & $\vartheta$ & $\checkmark$ & $\stackrel{p}{v}$ & 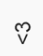 & $\stackrel{v}{v}$ & $\tilde{v}$ & $\stackrel{t}{v}$ & $\vartheta$ & 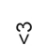 & 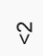 & 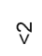 & $\checkmark$ & $\tilde{v}$ & v & $\stackrel{\mathbb{v}}{v}$ & $q$ & $\stackrel{p}{q}$ \\
\hline $8 \frac{5}{2}$ & ळे & $\overline{\grave{N}}$ & $\stackrel{\varphi}{v}$ & $\stackrel{\odot}{\vee}$ & N & N & $\tilde{v}$ & $\stackrel{9}{-}$ & $\stackrel{\infty}{\sim}$ & J & $\dddot{0}$ & $\stackrel{\infty}{q}$ & $\infty$ & 음 & $\stackrel{+}{\square}$ & $\stackrel{m}{m}$ & $\stackrel{\sim}{N}$ & ల్ల & 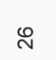 & ల్ల \\
\hline 잉 & 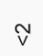 & $\stackrel{\tilde{v}}{\mathrm{v}}$ & $\vec{v}$ & $\dot{v}$ & $\stackrel{\sim}{~}$ & $\stackrel{\mathscr{N}}{\sim}$ & v & $\bar{v}$ & $\tilde{v}$ & $\stackrel{\vartheta}{v}$ & $\vec{v}$ & $v$ & 음 & $\stackrel{\infty}{\leftarrow}$ & $\stackrel{\leftrightarrow}{\sim}$ & $\widetilde{v}$ & $\bar{v}$ & $\underset{\nabla}{ }$ & 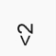 & $\mathrm{v}$ \\
\hline$\overline{\mathbf{m}} \mathbf{\mathrm { z }}$ & $\frac{\circ}{v}$ & $\stackrel{\text { ָิ }}{\mathrm{v}}$ & $\stackrel{\circ}{\stackrel{0}{v}}$ & 品 & 吊 & $\stackrel{\leftrightarrow}{v}$ & $\frac{\circ}{v}$ & $\frac{o}{v}$ & $\frac{\rho}{v}$ & จั & 음 & $\frac{\circ}{v}$ & $\stackrel{\circ}{\vee}$ & ᄋ & $\stackrel{\circ}{\circ}$ & $\frac{\rho}{v}$ & 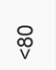 & ดे & $\frac{\circ}{v}$ & $\frac{O}{v}$ \\
\hline 舀 $\frac{\bar{z}}{2}$ & $\stackrel{\text { tे }}{\circ}$ & $\stackrel{\circ}{\circ}$ & $\hat{o}$ & $\stackrel{m}{\stackrel{m}{*}}$ & $\bar{v}$ & $\stackrel{0}{\stackrel{0}{*}}$ & r̃o & ָ̃ & 广্ণ & ró & ró & 品 & $\stackrel{\circ}{\leftarrow}$ & 多 & $\check{F}$ & O̊ & б. & $\stackrel{\circ}{i}$ & ำ & ó \\
\hline $\bar{\alpha} \frac{\bar{\partial}}{2}$ & $\stackrel{\circ}{v}$ & $\stackrel{\mathscr{L}}{\mathrm{v}}$ & $\stackrel{\nabla}{v}$ & $\stackrel{\vec{v}}{v}$ & $\stackrel{\vec{v}}{v}$ & $\stackrel{\vec{v}}{v}$ & $\stackrel{0}{\circ}$ & $\hat{v}$ & $\stackrel{\circ}{v}$ & $\frac{\mathscr{L}}{\mathrm{v}}$ & $\stackrel{\vec{v}}{v}$ & $\frac{O}{v}$ & 움 & $\stackrel{\mathcal{N}}{v}$ & $\stackrel{\sim}{\mathrm{v}}$ & $\frac{\circ}{v}$ & 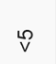 & 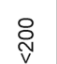 & $\hat{v}$ & $\hat{v}$ \\
\hline 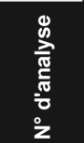 & 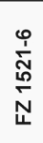 & 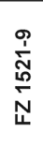 & $\begin{array}{l}\stackrel{N}{N} \\
\stackrel{N}{N} \\
\mathbb{N}\end{array}$ & 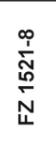 & 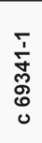 & 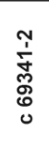 & 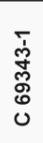 & $\begin{array}{l}\bar{\delta} \\
\bar{d} \\
0 \\
0 \\
0\end{array}$ & $\begin{array}{l}\text { 今. } \\
\text { ơ } \\
0 \\
0 \\
0\end{array}$ & 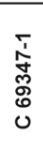 & 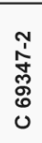 & $\begin{array}{l}\text { ? } \\
\text { ?े } \\
\text { o. } \\
0 \\
0\end{array}$ & 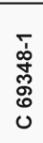 & $\begin{array}{l}\bar{g} \\
\text { go } \\
0 \\
0 \\
0\end{array}$ & $\begin{array}{l}\bar{\delta} \\
\text { O. } \\
00 \\
0 \\
0\end{array}$ & $\begin{array}{l}\bar{i} \\
\overline{0} \\
0 \\
0 \\
0\end{array}$ & $\begin{array}{l}\text { Tे } \\
\stackrel{0}{0} \\
0 \\
0 \\
0\end{array}$ & $\begin{array}{l}\bar{i} \\
\text { ¿. } \\
0 \\
0\end{array}$ & 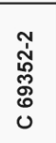 & 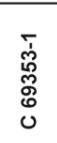 \\
\hline 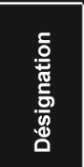 & 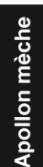 & 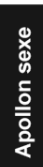 & 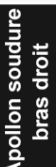 & 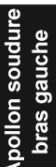 & 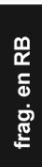 & 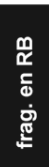 & 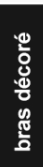 & 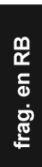 & 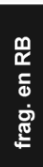 & 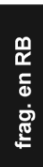 & 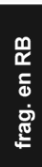 & 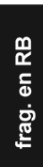 & 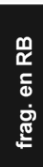 & 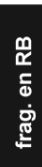 & 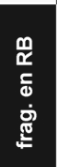 & 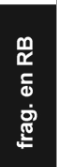 & 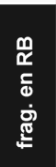 & 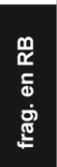 & 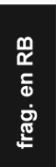 & 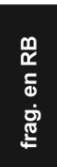 \\
\hline
\end{tabular}




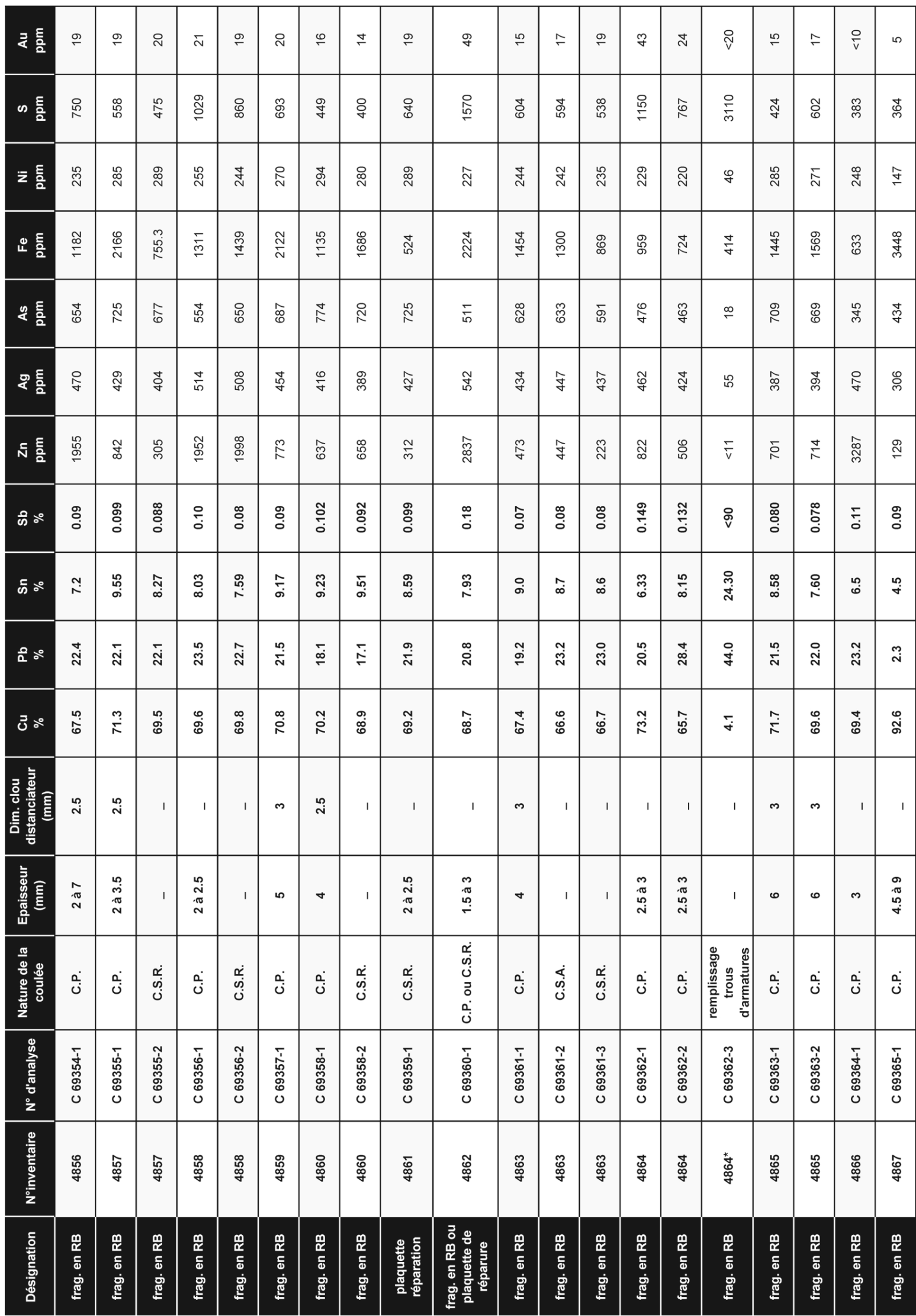




\begin{tabular}{|c|c|c|c|c|c|c|c|c|c|c|c|c|c|c|c|c|c|c|c|c|}
\hline$>\frac{E}{2}$ & $\stackrel{\circ}{\mathrm{N}}$ & v & $\bar{v}$ & $\tilde{v}$ & $\tilde{v}$ & $\stackrel{\circ}{\mathrm{i}}$ & $\tilde{v}$ & $m$ & - & $\stackrel{m}{i}$ & $\dot{v}$ & $\stackrel{\infty}{\sim}$ & $\stackrel{\odot}{i}$ & v & v & $\dot{v}$ & v & $\bar{v}$ & $\dot{v}$ & F \\
\hline$\supset \frac{\xi}{0}$ & $\stackrel{q}{v}$ & is & 畩 & ใู & Q & ஜे & ช్ & $\stackrel{R}{R}$ & . & $\stackrel{\sim}{v}$ & $\frac{8}{v}$ & $\frac{8}{v}$ & ใे & $\bar{\sigma}$ & 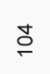 & 䖞 & 吕 & 8 & $\frac{8}{v}$ & $\stackrel{\mathscr{v}}{v}$ \\
\hline i= $\frac{5}{2}$ & $\bar{v}$ & $\checkmark$ & ఫ & $\stackrel{\text { ì }}{\text { s. }}$ & $\bar{v}$ & $\stackrel{\mathscr{N}}{\mathrm{N}}$ & $\widehat{v}$ & $\hat{v}$ & 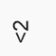 & $\bar{v}$ & v & $\stackrel{\circ}{\stackrel{\leftrightarrow}{r}}$ & $\bar{v}$ & t & v & $\stackrel{N}{v}$ & ป & $\tilde{v}$ & $\vartheta$ & $\bar{v}$ \\
\hline$\stackrel{\circ}{\circ} \frac{\bar{g}}{2}$ & $\stackrel{\phi}{v}$ & $\stackrel{g}{v}$ & $\tilde{\widetilde{v}}$ & $\stackrel{\circ}{v}$ & $\stackrel{\leftrightarrow}{v}$ & 品 & 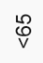 & ஜֶ & $\tilde{\tilde{v}}$ & $\stackrel{\sim}{v}$ & $\frac{8}{v}$ & 品 & : & fo & $\stackrel{\mathscr{Q}}{\mathrm{v}}$ & 吕 & of & ปิ & $\frac{8}{v}$ & $\stackrel{\sim}{v}$ \\
\hline ळ $\frac{\mathrm{g}}{2}$ & $\stackrel{\mathcal{V}}{v}$ & $\underset{v}{R}$ & $\stackrel{N}{N}$ & $\stackrel{\circ}{\mathrm{v}}$ & O্v & ᄋ్ల & 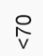 & $\underset{\substack{p \\
v}}{ }$ & f & $\frac{L}{v}$ & $\stackrel{8}{\vee}$ & Pे & ᄋ్ & 䎏 & $\stackrel{R}{v}$ & $\frac{i}{v}$ & 䎏 & $\stackrel{d}{\sim}$ & $\stackrel{\circ}{\vee}$ & $\frac{L}{v}$ \\
\hline a. $\frac{\xi}{2}$ & $\stackrel{\infty}{-}$ & $\stackrel{\mathscr{Q}}{\mathrm{V}}$ & $\stackrel{R}{v}$ & প్ల & ơ & $\stackrel{\phi}{v}$ & $\stackrel{\mathscr{m}}{v}$ & $\stackrel{\circ}{\circ}$ & $\stackrel{q}{v}$ & $\stackrel{\circ}{v}$ & 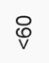 & g & $\hat{\iota}$ & $\stackrel{\mathscr{Q}}{\mathrm{V}}$ & $\frac{\mathscr{m}}{v}$ & 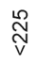 & 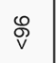 & \& & $\stackrel{\square}{\vee}$ & मे \\
\hline$\stackrel{\circ}{\Sigma} \frac{\bar{g}}{2}$ & $\dot{v}$ & প్ల & $\stackrel{\mathscr{N}}{\mathrm{V}}$ & $\hat{v}$ & $\widehat{v}$ & $\widehat{v}$ & : & 品 & $\stackrel{\sim}{v}$ & $\dot{v}$ & $\stackrel{\circ}{v}$ & $\hat{v}$ & $\hat{v}$ & P & 品 & $\underset{v}{O}$ & O্ & $\stackrel{n}{v}$ & $\frac{\vec{v}}{v}$ & مْ \\
\hline$\frac{c}{\frac{c}{\Sigma}} \frac{\bar{\partial}}{2}$ & $\bar{v}$ & $\checkmark$ & $\stackrel{\circ}{i}$ & $\stackrel{\nabla}{\rightleftarrows}$ & $\grave{i}$ & 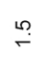 & $\stackrel{0}{\stackrel{0}{0}}$ & దొ & $\stackrel{\text { !n }}{\leftarrow}$ & 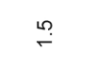 & v & $\hat{m}$ & $m$ & on & $\stackrel{\infty}{\substack{0 \\
\mathrm{v}}}$ & $\stackrel{m}{\bar{v}}$ & मे & ஸี & $\hat{m}$ & $\simeq$ \\
\hline$\frac{00}{2} \frac{5}{2}$ & $\hat{N}$ & $\bar{\sigma}_{\bar{v}}$ & $\bar{\sigma}_{\bar{v}}^{-}$ & 8 & مִ & $\stackrel{\sim}{\sim}$ & $\bar{\sigma}_{\bar{v}}$ & $\stackrel{0}{0}$ & $\bar{v}$ & $\bar{v}$ & న & $\stackrel{\circ}{N}$ & $\dot{t}$ & so & $\bar{v}^{\circ}$ & ć & $\bar{v}$ & $\bar{i}$ & $\kappa$ & $\tilde{N}$ \\
\hline$\leq \underline{\frac{\partial}{2}}$ & $\stackrel{\curvearrowleft}{V}$ & $\stackrel{\odot}{\square}$ & g & ס्र & $\stackrel{p}{v}$ & $\stackrel{\rho}{v}$ & $\underset{\substack{\infty \\
v}}{ }$ & $\underset{\substack{v \\
v}}{\infty}$ & f & $\stackrel{\circ}{V}$ & $\stackrel{\circ}{v}$ & $\stackrel{8}{v}$ & $\stackrel{O}{v}$ & $\stackrel{\circ}{v}$ & $\underset{\substack{\infty \\
\mathrm{D}}}{ }$ & $\frac{\mathscr{m}}{v}$ & $\stackrel{\circ}{\square}$ & $\stackrel{\mathscr{v}}{v}$ & $\stackrel{\circ}{\square}$ & $\stackrel{\circ}{v}$ \\
\hline 옾 흠 & 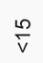 & $\stackrel{\mathscr{v}}{\tilde{v}}$ & $\stackrel{\infty}{v}$ & ס् & $\ddot{v}$ & $\stackrel{O}{v}$ & $\mathscr{v}$ & D্ & $\stackrel{\infty}{v}$ & $\stackrel{L}{V}$ & $\ddot{g}$ & $\stackrel{P}{\vartheta}$ & Oे & $\stackrel{\mathscr{V}}{v}$ & $\stackrel{\mathscr{V}}{\mathscr{V}}$ & 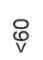 & $\mathscr{\varnothing}$ & $\stackrel{\tilde{V}}{\mathrm{~V}}$ & $\ddot{\widetilde{v}}$ & $\stackrel{\circ}{v}$ \\
\hline ن $\frac{E}{2}$ & $\vartheta$ & סे & 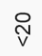 & $\stackrel{\circ}{v}$ & $\stackrel{\circ}{v}$ & $\frac{O}{v}$ & 跎 & ơ & $\stackrel{\vec{v}}{v}$ & $\vartheta$ & $\stackrel{\text { v }}{\mathrm{v}}$ & $\stackrel{\circ}{v}$ & $\stackrel{\varrho}{v}$ & 8 & ᄋ & $\stackrel{q}{v}$ & סे & $\stackrel{n}{v}$ & $\stackrel{\vec{v}}{v}$ & $\vartheta$ \\
\hline ¿ & $\stackrel{\infty}{\infty}$ & $\vec{v}$ & $\tilde{v}$ & a & v & $\widetilde{v}$ & $\vartheta$ & $\stackrel{\vartheta}{v}$ & 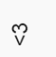 & 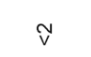 & $\mathrm{v}$ & $\stackrel{v}{v}$ & v & t & $\vartheta$ & Q & $\dot{v}$ & v & v & $\mathrm{v}$ \\
\hline 응 & N & ০ & $\stackrel{\text { S }}{N}$ & $\approx$ & $\bar{N}$ & $\stackrel{\mathcal{N}}{N}$ & F & $\mathscr{F}$ & $\stackrel{d}{N}$ & 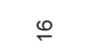 & d & $\stackrel{d}{N}$ & N & $\stackrel{m}{\sim}$ & $\stackrel{\text { N }}{\sim}$ & $\stackrel{\circ}{\circ}$ & \& & ร & Nָ & 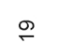 \\
\hline 엉 & 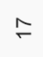 & v & v & 广 & 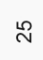 & $\stackrel{m}{\stackrel{2}{2}}$ & $\vec{v}$ & $\vec{v}$ & $\stackrel{v}{v}$ & in & $\stackrel{N}{\sim}$ & $\simeq$ & N & v & v & $\hat{v}$ & $\vartheta$ & $\bar{v}$ & $\stackrel{\circ}{\dot{m}}$ & $\stackrel{0}{\circ}$ \\
\hline$\overline{\mathrm{m}} \overline{\mathrm{D}}$ & $\approx$ & त् & $\stackrel{\text { Nิ }}{\mathrm{v}}$ & 号 & $\&$ & \& & 윰 & 읍 & $\frac{O}{V}$ & 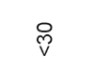 & $\frac{8}{v}$ & 品 & $\stackrel{\mathscr{v}}{v}$ & ָิ & 응 & 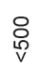 & ิㅗํ & $\frac{O}{v}$ & $\frac{o}{v}$ & סे \\
\hline 舀 $\frac{\bar{\partial}}{2}$ & $\bar{v}$ & ১. & ¿ & $\stackrel{\text { }}{~}$ & $\bar{v}$ & $\check{F}$ & g. & $\stackrel{\circ}{\mathrm{N}}$ & g. & $\bar{v}$ & $\stackrel{m}{\mathrm{~N}}$ & $\check{F}$ & $\bar{v}$ & $\stackrel{\circ}{-}$ & $\stackrel{\infty}{\infty}$ & $\stackrel{N}{\sim}$ & مُ & ś & $\overline{\mathrm{N}}$ & $\bar{v}$ \\
\hline < $\frac{E}{2}$ & $\stackrel{\circ}{\stackrel{\circ}{h}}$ & $\stackrel{\llcorner}{V}$ & $\frac{O}{v}$ & N & $\stackrel{\vec{v}}{\mathrm{v}}$ & จิ & $\stackrel{\vec{v}}{\mathrm{v}}$ & $\stackrel{\text { v }}{\mathrm{v}}$ & $\frac{\circ}{v}$ & $\stackrel{\circ}{v}$ & 8 & $\stackrel{\vec{v}}{v}$ & స్ & $\frac{n}{v}$ & $\stackrel{\nabla}{v}$ & $\stackrel{\stackrel{\rho}{ }}{\rightleftharpoons}$ & $\frac{n}{v}$ & $\widehat{v}$ & 哥 & $\stackrel{\simeq}{\simeq}$ \\
\hline$\stackrel{\mathscr{D}}{\stackrel{0}{2}}$ & $\begin{array}{l}\text { 咅 } \\
0 \\
0 \\
0 \\
0\end{array}$ & 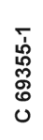 & 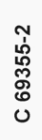 & $\begin{array}{l}\bar{\delta} \\
\stackrel{0}{0} \\
0 \\
0 \\
0\end{array}$ & $\begin{array}{l}\text { 今. } \\
\text { o. } \\
0 \\
0 \\
0\end{array}$ & $\begin{array}{l}\text { 离 } \\
0 \\
0 \\
0 \\
0\end{array}$ & 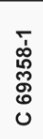 & 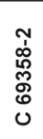 & 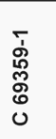 & $\begin{array}{l}\overline{\dot{d}} \\
\text { o. } \\
\text { ơ } \\
0\end{array}$ & $\begin{array}{l}\bar{I} \\
\overline{0} \\
0 \\
0 \\
0 \\
0\end{array}$ & 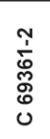 & $\begin{array}{l}\frac{m}{0} \\
\overline{0} \\
0 \\
0 \\
0\end{array}$ & 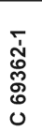 & 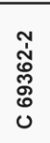 & 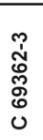 & $\begin{array}{l}\bar{\delta} \\
\overline{0} \\
0 \\
0 \\
0\end{array}$ & 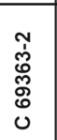 & $\begin{array}{l}\bar{y} \\
\mathbf{d} \\
0 \\
0 \\
0\end{array}$ & 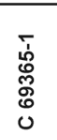 \\
\hline 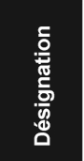 & 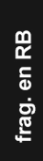 & 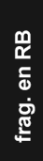 & 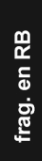 & 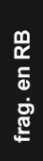 & 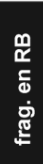 & 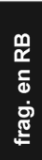 & 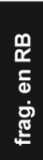 & 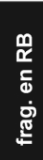 & 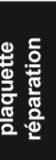 & 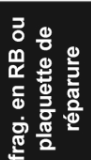 & 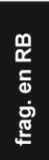 & 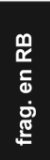 & 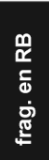 & 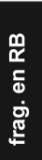 & 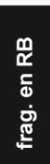 & 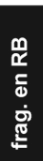 & 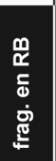 & 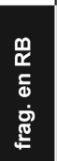 & 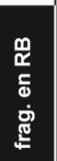 & 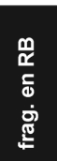 \\
\hline
\end{tabular}




\begin{tabular}{|c|c|c|c|c|c|c|c|c|c|c|c|c|c|c|c|c|c|c|c|c|}
\hline ₹ & $\stackrel{\square}{\square}$ & $\stackrel{9}{\sigma}$ & $\stackrel{f}{f}$ & N & \& & F & $\stackrel{m}{m}$ & $\bar{\sigma}$ & g & ले & $\hat{\omega}$ & 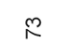 & $\stackrel{\infty}{-}$ & $\stackrel{5}{ \pm}$ & $\stackrel{\llcorner}{\stackrel{2}{2}}$ & $\stackrel{\llcorner}{\stackrel{2}{2}}$ & $\hat{N}$ & $\stackrel{\llcorner}{N}$ & $\stackrel{\infty}{\sim}$ & $\frac{N}{v}$ \\
\hline$\infty \frac{5}{2}$ & 守 & జี & $\underset{\substack{\infty \\
\mathrm{v}}}{ }$ & స్ & ֻั & 总 & 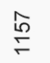 & 옹 & $\underset{⿱}{g}$ & 兽 & बे & $\stackrel{\mathscr{D}}{\sim}$ & 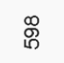 & $\stackrel{\frac{N}{V}}{V}$ & $\stackrel{\Re}{\stackrel{2}{2}}$ & 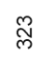 & హ్ & 㐫 & $\stackrel{\mathbb{ٌ}}{\stackrel{\sim}{\sim}}$ & ஜ্চু \\
\hline $\bar{z} \frac{E}{2}$ & 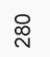 & స్ల్ & $\stackrel{\varphi}{m}$ & 占 & $\bar{N}$ & $\stackrel{\widehat{N}}{\mathrm{~N}}$ & $\stackrel{\infty}{\circ}$ & : P & $\stackrel{\infty}{\sim}$ & $\bar{N}$ & $\stackrel{\infty}{\sim}$ & $\stackrel{\infty}{\sim}$ & $\stackrel{\stackrel{g}{N}}{\sim}$ & 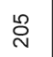 & 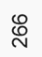 & N & : & 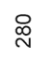 & 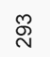 & $\stackrel{\infty}{\sim}$ \\
\hline 은 $\frac{\overline{2}}{2}$ & 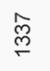 & $\infty$ & $\infty_{\infty}$ & $\bar{\sigma}$ & 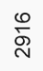 & 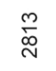 & $\frac{\pi}{\pi}$ & & $\stackrel{0}{\stackrel{2}{R}}$ & 䌸 & $\begin{array}{l}\mathscr{8} \\
\stackrel{\circ}{\square}\end{array}$ & $R$ & 䓀 & 涡 & : & 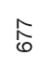 & 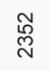 & 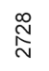 & 总 & \&্ল \\
\hline \& $\frac{E}{2}$ & 志 & $\stackrel{8}{q}$ & $\underset{\tilde{V}}{\tilde{V}}$ & 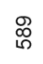 & 욶 & 点 & 总 & $\stackrel{9}{5}$ & 芯 & 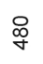 & : & 总 & ิิ & 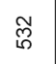 & 怘 & స్ & О్ & స్ & 占 & 寽 \\
\hline \& & $\stackrel{\mathscr{Q}}{+}$ & 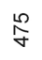 & 案 & 总 & $\stackrel{\infty}{5}$ & $\stackrel{\mathscr{్}}{\circ}$ & 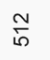 & $\frac{d}{\mathcal{N}}$ & $q$ & 昌 & : & 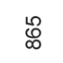 & $\stackrel{\infty}{\text { \& }}$ & $\stackrel{\mathscr{G}}{\stackrel{\leftrightarrow}{2}}$ & 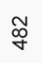 & 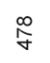 & 通 & $\underset{్}{ }$ & 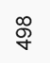 & $\stackrel{\sim ్}{\sim}$ \\
\hline 동 & 总 & मे & $\stackrel{\text { I }}{ }$ & $\stackrel{\text { N }}{ }$ & 䒺 & 兽 & $\frac{m}{6}$ & बু & ্ֶֻٍ & $\underset{\infty}{\tilde{D}}$ & ల్ల & $\stackrel{\mathbb{N}}{\mathbb{N}}$ & $\stackrel{\leftrightarrow}{\circledR}$ & $\hat{v}$ & న్ & ְొ & 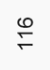 & $\stackrel{\oplus}{\leftarrow}$ & $\stackrel{\circ}{\circ}$ & ㅇ \\
\hline के ०० & $\stackrel{8}{\circ}$ & o̊ & $\begin{array}{l}\circ \\
\stackrel{\circ}{0}\end{array}$ & $\stackrel{\text { N }}{\circ}$ & $\stackrel{0}{\circ}$ & $\stackrel{\infty}{0}$ & $\stackrel{N}{0}$ & $\stackrel{\circ}{\circ}$ & $\frac{\bar{\alpha}}{\stackrel{\infty}{0}}$ & $\stackrel{n}{0}$ & $\stackrel{0}{0}$ & $\stackrel{5}{0}$ & $\stackrel{8}{\circ}$ & 总 & $\frac{\swarrow}{\circ}$ & ò & $\stackrel{m}{\frac{m}{p}}$ & $\frac{0}{0}$ & 움 & $\frac{0}{0}$ \\
\hline ఱ & $\hat{i}$ & $\stackrel{m}{+}$ & $\stackrel{f}{+}$ & $\stackrel{\infty}{\omega}$ & $\stackrel{\circ}{\infty}$ & $\stackrel{\wp}{\infty}$ & $\stackrel{\circ}{\circ}$ & $\stackrel{\substack{\infty \\
\infty}}{\infty}$ & 吕 & $\underset{\infty}{\infty}$ & $\stackrel{\text { İ }}{\text { İ }}$ & $\stackrel{\circ}{\dot{\sigma}}$ & $\underset{\infty}{\square}$ & ָ̃ & $\begin{array}{c}\infty \\
\substack{\infty \\
\infty \\
\infty}\end{array}$ & 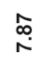 & $\underset{\infty}{\widetilde{\infty}}$ & 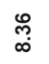 & ஓ̊. & @్ల్ \\
\hline$\frac{\circ}{2} \circ \circ$ & 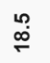 & $\stackrel{m}{\sigma}$ & $\stackrel{\circ}{\circ}$ & $\stackrel{\widetilde{N}}{\stackrel{N}{\sim}}$ & ஸें & $\stackrel{\Re}{\check{r}}$ & ঙี่ & $\stackrel{m}{g}$ & $\stackrel{\infty}{\stackrel{\infty}{\infty}}$ & స̃ & 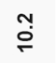 & $\stackrel{m}{+}$ & ָे & 吊 & $\stackrel{\vec{N}}{\dot{N}}$ & $\overline{\tilde{\sim}}$ & $\stackrel{\text { in }}{\text { D. }}$ & $\dot{\vec{N}}$ & $\stackrel{\sigma}{\rho}$ & 웅 \\
\hline$\overline{0}$ ठ̊ & N & 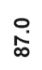 & $\begin{array}{l}\mathscr{8} \\
\dot{\infty} \\
\infty\end{array}$ & 㐫 & 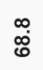 & Ñ & $\widehat{\widehat{o s}}$ & $\bar{N}$ & $\hat{\hat{N}}$ & $\stackrel{0}{\leftrightarrow}$ & $\stackrel{\infty}{R}$ & ๙ู่ & $\bar{\phi}$ & 免 & $\underset{\Sigma}{\Sigma}$ & $\underset{\mathscr{\infty}}{\mathfrak{n}}$ & 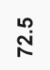 & $\stackrel{0}{0}$ & 官 & $\stackrel{\infty}{\stackrel{2}{R}}$ \\
\hline 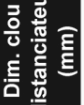 & $\infty$ & I & I & I & $\sim$ & 1 & 1 & 1 & 1 & I & 1 & 1 & 1 & 1 & $\stackrel{\sim}{N}$ & 1 & $\stackrel{\sim}{N}$ & 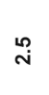 & 1 & \\
\hline $\begin{array}{l}\text { 总 } \\
\text { 总 } \\
\text { 兽 }\end{array}$ & I & 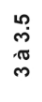 & I & 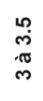 & $\underset{\sim}{\infty}$ & 1 & D. & $\infty$ & $m$ & $\stackrel{\substack{\pi \\
\sim}}{\sim}$ & 赵 & 1 & 1 & 1 & 萬 & 1 & $\stackrel{\sim}{N}$ & $\stackrel{\text { N }}{\text { N }}$ & I & \\
\hline 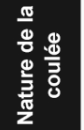 & ن̀ & نे & 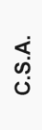 & ن. & i. & 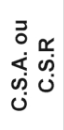 & í & i̊ & ن. & ن̀ & نें & 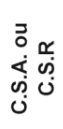 & 峁 & ن̀ं & ì & $\begin{array}{l}0 \\
0 \\
0 \\
0 \\
0 \\
0\end{array}$ & ن. & : & 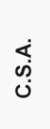 & $\begin{array}{l}\stackrel{\alpha}{\sigma j} \\
\stackrel{j}{j} \\
\dot{\omega}\end{array}$ \\
\hline 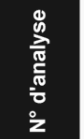 & $\begin{array}{l}\overline{\dot{\delta}} \\
\stackrel{0}{0} \\
\stackrel{0}{0}\end{array}$ & $\begin{array}{l}\text { ī } \\
0.00 \\
0 \\
0 \\
0\end{array}$ & $\begin{array}{l}\text { ₹ } \\
0 \\
0 \\
0 \\
0 \\
0\end{array}$ & $\begin{array}{l}\bar{\alpha} \\
0.0 \\
00 \\
0 \\
0 \\
0\end{array}$ & $\begin{array}{l}\bar{\delta} \\
0 \\
0 \\
0 \\
0 \\
0\end{array}$ & 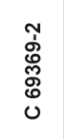 & $\begin{array}{l}\text { ò } \\
\text { ô. } \\
0 \\
0\end{array}$ & $\begin{array}{l}\frac{\bar{T}}{\hat{i}} \\
0.00 \\
0 \\
0\end{array}$ & 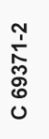 & $\begin{array}{l}\text { ì } \\
\text { N. } \\
0 \\
0 \\
0\end{array}$ & 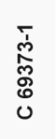 & 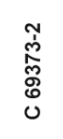 & $\begin{array}{l}\text { 융 } \\
00 \\
0 \\
0\end{array}$ & $\begin{array}{l}\overline{1} \\
\text { 今. } \\
0 \\
0 \\
0\end{array}$ & $\begin{array}{l}\text { 它 } \\
\stackrel{\circ}{\circ} \\
0 \\
0 \\
0\end{array}$ & 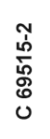 & $\begin{array}{l}\overline{\dot{0}} \\
\dot{\circ} \\
0 \\
0\end{array}$ & 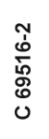 & \begin{tabular}{l}
3 \\
\multirow{0}{0}{} \\
$\dot{0}$ \\
0 \\
0 \\
0
\end{tabular} & 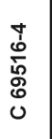 \\
\hline . & : & $\underset{\substack{\infty \\
\stackrel{\alpha}{d}}}{\mathbb{2}}$ & 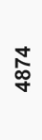 & 点 & 总 & 总 & 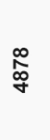 & $\begin{array}{l}\bar{\alpha} \\
\stackrel{\alpha}{\sigma}\end{array}$ & $\begin{array}{l}\overline{\mathbf{o}} \\
\stackrel{\sigma}{\alpha}\end{array}$ & 离 & 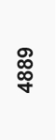 & 兽 & 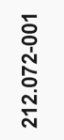 & $\begin{array}{l}\bar{o} \\
\dot{\infty} \\
\stackrel{\leftrightarrow}{\text { Sे }} \\
\stackrel{N}{N}\end{array}$ & 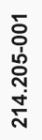 & 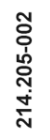 & 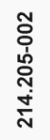 & 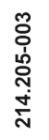 & 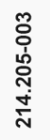 & \\
\hline 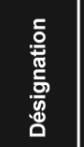 & 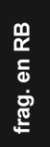 & 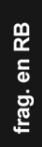 & 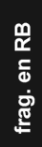 & 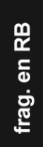 & 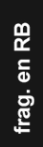 & 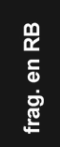 & 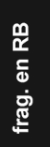 & 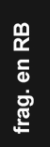 & 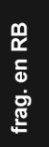 & 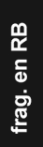 & 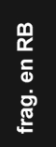 & 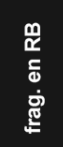 & 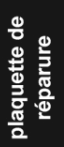 & 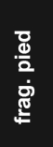 & 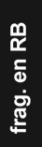 & 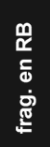 & 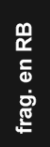 & 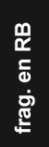 & 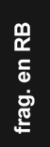 & \\
\hline
\end{tabular}




\begin{tabular}{|c|c|c|c|c|c|c|c|c|c|c|c|c|c|c|c|c|c|c|c|c|}
\hline$>\frac{5}{2}$ & m & $m$ & $\bar{v}$ & $\grave{i}$ & $\dot{v}$ & $\grave{i}$ & $v$ & $\bar{v}$ & 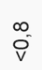 & M̀ & $\hat{N}$ & ֶָ & $\stackrel{M}{N}$ & $\tilde{v}$ & $\tilde{v}$ & $\bar{v}$ & $\bar{v}$ & $\bar{v}$ & $\stackrel{\infty}{i}$ & 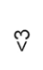 \\
\hline כ & $\stackrel{\mathscr{V}}{.}$ & $\stackrel{8}{\square}$ & $\stackrel{\substack{m \\
v}}{v}$ & $\stackrel{\odot}{\square}$ & $\frac{8}{v}$ & ஜे & ధి & 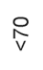 & P. & 㝏 & 吕 & $\widetilde{0}$ & Pे & $\stackrel{ }{\stackrel{D}{v}}$ & $\frac{\mathscr{O}}{\mathrm{v}}$ & $\stackrel{\circ}{\mathrm{v}}$ & $\stackrel{i}{v}$ & 8 & $\stackrel{8}{\mathrm{~V}}$ & 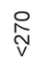 \\
\hline$=\frac{5}{\partial}$ & $\stackrel{\leftrightarrow}{\leftarrow}$ & $\bar{v}$ & $\stackrel{v}{v}$ & $\bar{v}$ & $v$ & $\stackrel{+}{\leftarrow}$ & $\stackrel{n}{N}$ & v & v & $\bar{v}$ & $\stackrel{\infty}{\leftarrow}$ & $\stackrel{\sim}{\square}$ & $\tilde{v}$ & $\widehat{v}$ & $\hat{v}$ & $\checkmark$ & $\tilde{v}$ & v & $\widetilde{v}$ & $\hat{v}$ \\
\hline$\stackrel{\circ}{\circ} \frac{\bar{\partial}}{2}$ & $\stackrel{\mathscr{Q}}{\mathrm{V}}$ & প్ v & $\tilde{\widetilde{v}}$ & $\stackrel{p}{v}$ & $\frac{o}{v}$ & ధి & ధి & $\stackrel{\omega}{v}$ & $\tilde{\widetilde{v}}$ & ใे & ดे & Oे & Pे & $\stackrel{\mathscr{\sigma}}{\sigma}$ & ơ & $\tilde{\tilde{v}}$ & $\frac{O}{v}$ & ฟ & $\tilde{\tilde{v}}$ & $\stackrel{\leftrightarrow}{Q}$ \\
\hline ळ & $\ddot{\nabla}$ & $\stackrel{n}{v}$ & $=$ & $\stackrel{\varrho}{v}$ & $\stackrel{\circ}{\checkmark}$ & $\stackrel{p}{v}$ & $\ddot{p}$ & $\stackrel{\infty}{v}$ & g & $\stackrel{\circ}{\mathrm{v}}$ & סे & $\frac{\mathscr{L}}{v}$ & $\ddot{p}$ & $\stackrel{\mathcal{N}}{\mathrm{v}}$ & 总 & ले & $\stackrel{\infty}{v}$ & 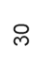 & g & $\underset{v}{q}$ \\
\hline a $\frac{\bar{z}}{\frac{2}{2}}$ & $\stackrel{\infty}{\square}$ & $\stackrel{n}{v}$ & $\stackrel{P}{v}$ & $\stackrel{\omega}{v}$ & $\stackrel{\circ}{\checkmark}$ & ซ & $\stackrel{p}{v}$ & $\stackrel{?}{v}$ & $\stackrel{p}{v}$ & $\stackrel{p}{v}$ & $\ddot{\nabla}$ & $\frac{n}{v}$ & ק्p & $\stackrel{\infty}{v}$ & $\stackrel{\substack{\mathrm{V} \\
\mathrm{v}}}{ }$ & $\stackrel{i}{v}$ & is & fे & $\stackrel{p}{v}$ & $\stackrel{\mathscr{m}}{v}$ \\
\hline 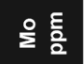 & $\hat{v}$ & $\vec{v}$ & $\stackrel{\mathscr{\sim}}{\sim}$ & $\vec{v}$ & $\stackrel{n}{v}$ & $\hat{v}$ & $\widehat{v}$ & $\stackrel{\circ}{v}$ & $\stackrel{\sim}{v}$ & $\widehat{v}$ & $\widehat{v}$ & tे & $\widehat{v}$ & 品 & $\stackrel{\varnothing}{\nabla}$ & $\stackrel{n}{v}$ & $\frac{n}{v}$ & $\stackrel{n}{v}$ & $\stackrel{\mathscr{N}}{\sim}$ & 导 \\
\hline$\frac{\varepsilon}{\Sigma} \frac{\bar{\partial}}{2}$ & 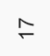 & $\bar{v}$ & - & $\bar{v}$ & $\stackrel{i}{i}$ & $\stackrel{m}{i}$ & مُ & $\hat{m}$ & $\stackrel{\circ}{\dot{m}}$ & $\stackrel{\leftrightarrow}{-}$ & $\stackrel{g}{\dot{q}}$ & $\bar{v}$ & $\bar{v}$ & $\simeq$ & $\stackrel{m}{\infty}$ & $\stackrel{\infty}{\wedge}$ & $\stackrel{+}{+}$ & $\bar{N}$ & $\stackrel{\curvearrowleft}{0}$ & $m$ \\
\hline$\frac{\text { 일 } \frac{\overline{2}}{2}}{2}$ & $\stackrel{\overbrace{}}{\sim}$ & $\bar{v}$ & $\bar{v}_{\bar{v}}$ & $\bar{v}$ & $\checkmark$ & $\stackrel{m}{=}$ & $\bar{v}$ & ó & $\overline{\dot{v}}$ & $\stackrel{\mathfrak{N}}{\stackrel{N}{N}}$ & $\bar{v}$ & $\stackrel{\text { ì }}{ }$ & $\bar{v}$ & $\stackrel{\nabla}{\sigma}$ & $\overline{\dot{v}}$ & $\bar{\sigma}$ & $\bar{q}_{\bar{v}}$ & $\bar{q}$ & $\bar{v}_{\bar{v}}$ & í \\
\hline$\leq \underline{\frac{\partial}{2}}$ & $\ddot{v}$ & $\stackrel{n}{V}$ & q & $\stackrel{\circ}{v}$ & $\stackrel{\circ}{\circ}$ & $\stackrel{\mathscr{v}}{v}$ & $\ddot{v}$ & $\stackrel{\rho}{\tilde{v}}$ & ㅇv & 它 & סे & $\frac{\mathscr{L}}{v}$ & $\ddot{\vartheta}$ & $\underset{\substack{\infty \\
v}}{\infty}$ & $\stackrel{\circ}{\square}$ & g & $\stackrel{\vec{v}}{v}$ & $\ddot{\tilde{v}}$ & g & 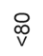 \\
\hline 옫흘 & $\ddot{\nabla}$ & $\stackrel{n}{v}$ & $\frac{\infty}{v}$ & $\stackrel{\circ}{v}$ & $\ddot{\vartheta}$ & $\stackrel{\text { V }}{\text { val }}$ & 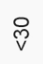 & $\frac{N}{v}$ & $\stackrel{\infty}{v}$ & 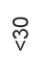 & $\ddot{\vartheta}$ & $\frac{\mathscr{L}}{v}$ & $\ddot{v}$ & $\stackrel{\mathscr{v}}{\mathscr{v}}$ & $\stackrel{\sim}{v}$ & $\stackrel{\infty}{v}$ & v & $\hat{\omega}$ & $\stackrel{\infty}{v}$ & $\underset{\mathscr{v}}{\mathscr{v}}$ \\
\hline$\stackrel{0}{0} \frac{\bar{g}}{2}$ & $\stackrel{\circ}{v}$ & $\stackrel{\vartheta}{v}$ & 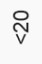 & $\stackrel{\varphi}{v}$ & $\stackrel{\vec{v}}{v}$ & $\stackrel{\varphi}{\vee}$ & $\stackrel{\circ}{v}$ & $\stackrel{\circ}{v}$ & $\stackrel{i}{v}$ & $\stackrel{\circ}{v}$ & $\stackrel{\circ}{v}$ & $\stackrel{\varphi}{\vee}$ & $\stackrel{\circ}{v}$ & g & $\stackrel{8}{\varnothing}$ & $\frac{n}{v}$ & $\stackrel{\circ}{v}$ & $\stackrel{\varrho}{v}$ & $\stackrel{\vec{v}}{v_{0}}$ & g v \\
\hline ¿ & v & v & $q$ & v & v & v & v & v & $\tilde{v}$ & v & v & v & $\stackrel{v}{v}$ & $\stackrel{\varphi}{v}$ & $\dot{v}$ & $\tilde{v}$ & $\tilde{v}$ & v & v & $\stackrel{\vartheta}{v}$ \\
\hline 응 & $\stackrel{\mathscr{N}}{ }$ & $\stackrel{q}{\dot{q}}$ & $\hat{m}$ & $\stackrel{\circ}{+}$ & $\simeq$ & $\stackrel{\circ}{\circ}$ & 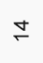 & $\begin{array}{l}\stackrel{0}{\rho} \\
\stackrel{\rho}{\longrightarrow}\end{array}$ & $\stackrel{m}{=}$ & $\stackrel{\square}{\square}$ & 우 & $\widetilde{v}$ & $\approx$ & $\nabla$ & q & $\hat{\lambda}$ & $\stackrel{\circ}{\circ}$ & $\stackrel{\circ}{\llcorner}$ & $\simeq$ & î \\
\hline 잉 & $\stackrel{?}{\stackrel{9}{2}}$ & $\stackrel{\infty}{-}$ & v & $\stackrel{\sim}{N}$ & 우 & $\bar{N}$ & $\stackrel{0}{\circ}$ & $\underset{\infty}{+}$ & v & $\stackrel{\circ}{i}$ & $\stackrel{N}{N}$ & $\stackrel{\infty}{\wedge}$ & $=$ & $F$ & 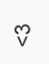 & v & $\bar{v}$ & v & v & $\dot{v}$ \\
\hline$\overline{\bar{\infty}} \overline{\mathrm{g}}$ & む & পి & $\frac{\circ}{v}$ & q & $\frac{8}{v}$ & $\stackrel{\mathscr{v}}{\vartheta}$ & Q & $\underset{\text { D }}{\infty}$ & $\frac{\rho}{v}$ & 它 & ดे & ల్ల & จे & 음 & ิㅗำ & $\frac{\rho}{v}$ & $\underset{\vee}{\otimes}$ & 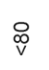 & $\frac{\circ}{v}$ & 음 \\
\hline 临 $\frac{\mathrm{z}}{2}$ & $\stackrel{\leftrightarrow}{\leftarrow}$ & $\bar{v}$ & 웅 & $\bar{v}$ & $r$ & 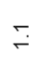 & $\bar{v}$ & $\tilde{O}$ & '̄ & $\bar{v}$ & $\bar{v}$ & 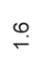 & $\bar{v}$ & $\stackrel{m}{N}$ & $\stackrel{\circ}{\circ}$ & $\stackrel{2}{0}$ & $\stackrel{\circ}{\dot{r}}$ & مò & $\stackrel{t}{0}$ & $\bar{i}$ \\
\hline $\bar{\alpha} \frac{\mathrm{g}}{2}$ & $\grave{\grave{N}}$ & $\stackrel{\circ}{v}$ & $\stackrel{\circ}{v}$ & N & $\stackrel{i}{v}$ & $\stackrel{\circledast 0}{\sigma}$ & $\stackrel{i}{v}$ & $\stackrel{\varphi}{v}$ & $\widehat{v}$ & $\hat{\infty}$ & $\stackrel{\nabla}{v}$ & \pm & $\stackrel{\vec{v}}{v}$ & 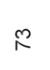 & $\frac{n}{V}$ & $\hat{v}$ & $\vartheta$ & $\hat{v}$ & $\stackrel{\circ}{v}$ & $\stackrel{\text { จิ }}{\text { s. }}$ \\
\hline$\stackrel{0}{0}$ & $\begin{array}{l}\bar{\delta} \\
\overline{0} \\
. \\
0\end{array}$ & $\begin{array}{l}\text { ĩ } \\
0 \\
0 \\
0 \\
0\end{array}$ & 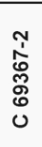 & $\begin{array}{l}\bar{\alpha} \\
\text { o. } \\
0 \\
0 \\
0\end{array}$ & $\begin{array}{l}\bar{\delta} \\
\text { o. } \\
0 \\
0 \\
0\end{array}$ & 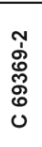 & 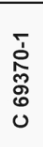 & 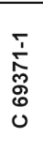 & 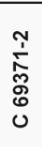 & 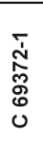 & 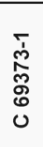 & 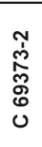 & $\begin{array}{l}0 \\
0 \\
0 \\
0 \\
0 \\
0\end{array}$ & $\begin{array}{l}\overline{1} \\
\stackrel{1}{0} \\
0 \\
0 \\
0\end{array}$ & 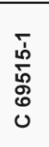 & 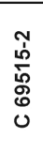 & $\begin{array}{l}\bar{\delta} \\
\stackrel{0}{0} \\
0 \\
0\end{array}$ & 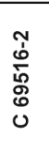 & 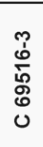 & 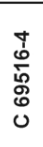 \\
\hline $\begin{array}{l}\frac{3}{7} \\
\frac{0}{0}\end{array}$ & 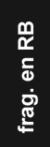 & 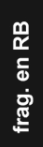 & 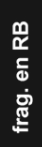 & 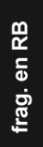 & 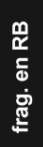 & 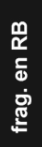 & 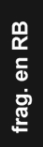 & 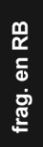 & 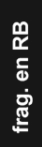 & 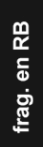 & 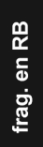 & 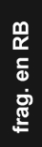 & 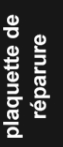 & 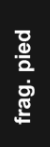 & 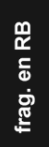 & 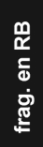 & 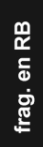 & 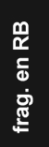 & 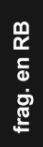 & 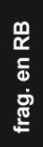 \\
\hline
\end{tabular}

